\title{
A General Semi-Markov Model for Coupled Lifetimes
}

\author{
Min $\mathrm{Ji}^{1}$ and Rui Zhou ${ }^{2}$ \\ ${ }^{1}$ Department of Mathematics, Towson University \\ ${ }^{2}$ Centre for Actuarial Studies, Department of Economics, University of Melbourne
}

June 2, 2018

\begin{abstract}
Joint life annuities with high last survivor benefit play an important role in the optimal annuity portfolio for a retired couple. The dependence between coupled lifetimes is crucial for valuing joint life annuities. Existing bivariate modelling of coupled lifetimes is based on outdated data with limited observation periods and does not take into account mortality improvement. In this paper, we propose a transparent and dynamic framework for modelling coupled lifetime dependence caused by both marital status and common mortality improvement factors. Dependence due to marital status is captured by a semi-Markov joint life model. Dependence due to common mortality improvement, which represents the correlation between mortality improvement patterns of coupled lives, is incorporated by a two-population mortality improvement model. The proposed model is applied to pricing the longevity risk in last survivor annuities sold in the US and the UK.
\end{abstract}

Keywords: Bivariate lifetime; Semi-Markov model; Longevity risk; Single life annuity; Last survivor annuity

\section{Introduction}

A recent study by Hubener et al. (2013) using a life cycle consumption and portfolio choice model showed that joint life annuities with high last survivor benefit account for a large portion of optimal annuity portfolios for retired couples. Dependence between coupled lives has been well-recognized (see Young et al., 1963; Cox \& Ford; 1964, Parkes et al., 1969; Frees et al., 1996; Youn \& Shemyakin, 2001; Ji et al., 2011; Spreeuw \& Owadally, 2013). Ji et al. (2011) has shown that dependence between coupled lives is an important factor 
in pricing joint life annuities. However, there has been little focus on stochastic bivariate modelling of coupled lifetimes. Insurance practice conventionally assumes independence between coupled lives for computational convenience. Another critical factor for valuing joint life annuities is the correlation between male and female mortality improvement. Current bivariate modelling of coupled lifetimes is based on outdated data with limited observation periods and does not take into account mortality improvement.

In this paper, we propose a stochastic bivariate model for dependent and dynamic modelling of coupled lifetimes. This approach is comprised of two parts: a semi-Markov multi-state model and a two-population mortality improvement model. This approach allows for the adaption of different assumptions of dependence between coupled lives and mortality improvement patterns.

The proposed semi-Markov model is an extension of the models introduced by Norberg (1989) and Ji et al. (2011). The former model used a four-state continuous-time Markov chain and focused on the long-term dependence effect. Ji et al. (2011) extended Norberg's model by introducing a semi-Markov model where the diminishing of bereavement effect was controlled by an exponentially decreasing function. However, there has been no sufficient data to justify the exponential decay pattern. Spreeuw and Owadally (2013) argued that a reverse sigmoid curve, which has a flat slope in early widowhood, might be more appropriate than an exponential decay function for modelling the bereavement effect. They intuitively believed that the speed at which the bereavement effect diminishes is initially slow.

In our semi-Markov multi-state model, the four state-dependent forces of mortality that control the transition between states are proportional to the corresponding individual marginal force of mortality. This is analogous to the Cox proportional hazards model. The proportional multiplicative factors in the married status are non-level age-dependent constants, which are less than one to ensure that the forces of mortality in the married status are lower than the marginal forces of mortality. The proportion in the widowed status is a decreasing function of the sojourn time in widowhood, hence modelling the decaying pattern of bereavement effect. The function initially increases the force of mortality of the surviving spouse due to the "broken-heart" effect which fades as time elapses and finally tapers off. We test three different functions for the bereavement effect: an exponential function, a Gaussian curve, and a reverse sigmoid function.

The calibration of the semi-Markov joint life model is attempted without raw mortality data by matching the marginal distribution of individual lifetimes to the mortality rates in a chosen life table, such as the annuity life table RP-2014 in the US and the S2 Series of Mortality Table in the UK. The marginal distributions of the semi-Markov model are calculated as the mixture of conditional survival probabilities with mixture weights based 
on the census data of populations in varying states of marital status. The three calibrated functions for bereavement effect have significantly different initial increases in the force of mortality at the death of a spouse and dramatically different speeds at which the bereavement effect tapers off. The assumption of exponential decay of bereavement has the highest initial value and the fastest decaying rate, while both the reverse sigmoid function and the Gaussian functions have more moderate initial value and diminishing speed than the exponential function.

We then incorporate a two-population mortality improvement model to the semi-Markov joint life model to model the time-varying dependence between the future lifetimes of coupled lives. Since male and female mortality improvements are highly correlated, it is necessary to model them jointly by a multi-population mortality model such that their correlations are adequately captured. Multi-population mortality models have been comprehensively studied (see Li \& Hardy, 2011; Cairns et al., 2011; Dowd et al., 2011; Zhou et al., 2014). In this paper, we choose a two-population Age-Period-Cohort (APC) model due to its robustness in mortality projection and follow Zhou et al. (2014) to model the male and female period effects correlatively by a bivariate random walk with drift.

We use the male and female mortality data in the age range of 50 to 95 in the past 50 years to calibrate the two-population mortality improvement model for the US and UK respectively. This range of data, which is believed to give reliable projection of future mortality paths, includes all the cohort parameters required for projecting future mortality improvement rates. Assuming annuitants experience the same mortality improvement as the general population, we combine the calibrated two-population APC model with the semi-Markov model to generate the dynamics of future lifetimes for coupled lives.

The two parts of the proposed model are calibrated separately due to the lack of longitudinal bivariate mortality data. Ideally, the semi-Markov model should be calibrated to a life insurance company's joint life data. However, the currently available bivariate mortality data is obsolescent. The proposed estimation method circumvents the need of bivariate data by matching the marginal survival probabilities of the semi-Markov model to the chosen annuity life table. The proposed estimation method can also be applied to bivariate mortality data if available.

The proposed model is applied to evaluate the risk premium of longevity risk in both single life and joint life annuities. The price of an annuity is evaluated as the expected present value of annuity payments under a risk-neutral probability measure. In a complete market, there is a unique risk-neutral probability measure. However, the annuity market is far from complete. Following Wang (2007) and Cox et al. (2006), we employ the multivariate exponential tilting method to derive a "market consistent" risk-neutral measure and therein 
implied risk premium based on annuity quotes in the current US and UK private annuity markets. In particular, we quote single life annuities and last survivor annuities and then determine the implied market price of longevity risk in the quoted annuities by equating the simulated prices under risk-neutral measure to the quoted market prices. Our goal is to compare the implied risk premiums of longevity risk in various annuities and examine the current market's view of longevity risk.

The remainder of this paper proceeds as follows. Section 2 introduces a general framework of semi-Markov mortality model for coupled lives and proposes a calibration method that circumvents the lack of bivariate mortality data. Section 3 describes the two-population APC mortality model and the calibration results using the US and UK mortality data. In section 4, the proposed model is applied to evaluate the implied US and UK market prices of longevity risk in various annuities using the normalized multivariate exponential tilting method. In section 5 we draw concluding remarks about our proposed model and findings.

\section{The Semi-Markov Joint life Model}

\subsection{The Model}

Let $(x)$ and $(y)$ denote coupled lives aged $x$ and $y$ respectively. Assume that $(x)$ is a husband and $(y)$ is a wife. $T_{x}$ and $T_{y}$ are their individual future lifetime random variables.

$\mu_{x+t}^{(1)}$ and $\mu_{y+t}^{(2)}$ define the marginal forces of mortality at future time $t$ (age $x+t$ and $y+t$ by then respectively) associated with the marginal distribution of $T_{x}$ and $T_{y}$. Here $x$ and $y$ are fixed and $t$ is a variable.

The model uses four states to describe the survival status of coupled lives: both are alive (state 0 ); only $(y)$ is alive (state 1 ); only $(x)$ is alive (state 2 ); and both are dead (state 3 ). The joint distribution of $T_{x}$ and $T_{y}$ is modeled by a semi-Markov model, with states and forces of transition shown in Figure 1.

The forces of transitions, $\mu_{x+t}^{01}, \mu_{y+t}^{02}, \mu_{x+t, s}^{23}$, and $\mu_{y+t, s}^{13}$ can be viewed as conditional forces of mortality. Here we ignore remarriage. $\mu_{x+t}^{01}$ and $\mu_{y+t}^{02}$ are the conditional forces of mortality for $(x)$ and $(y)$ at time $t$ respectively, conditional on the survival of their spouse but not on the age of their spouse. $\mu_{x+t, s}^{23}$ and $\mu_{y+t, s}^{13}$ are the conditional forces of mortality for $(x)$ and $(y)$ at time $t$ respectively, conditional on having stayed in the widowhood for $s$ years. The forces of transitions are defined to preserve the semi-Markov property, under which the probabilities of future death events are determined by the current state and how long the process has been in the current state.

All four conditional forces of mortality are proportional to the corresponding individual 


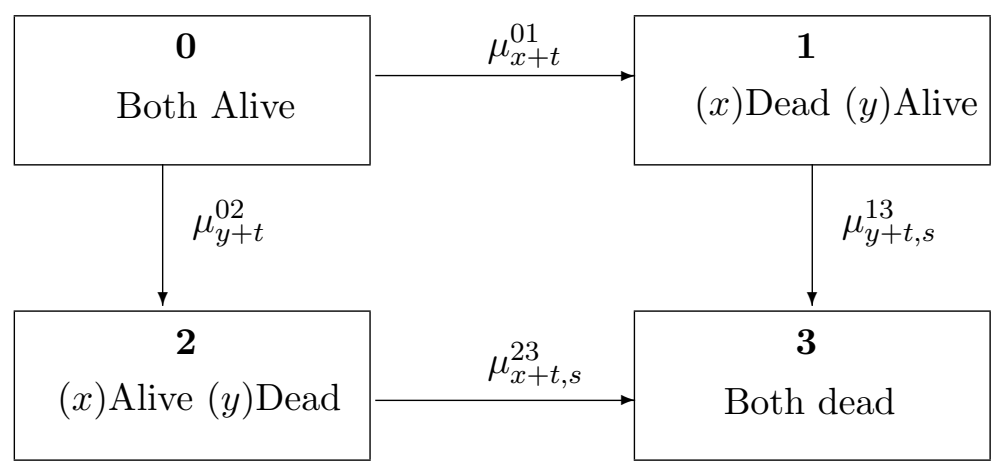

Figure 1: Semi-Markov Joint Life Model.

marginal forces of mortality. Expressed Mathematically,

$$
\begin{aligned}
\mu_{x+t}^{01} & =\psi_{1, x+t} \cdot \mu_{x+t}^{(1)}, \\
\mu_{y+t}^{02} & =\psi_{2, y+t} \cdot \mu_{y+t}^{(2)}, \\
\mu_{x+t, s}^{23} & =f_{1}(s) \cdot \mu_{x+t}^{(1)}, \\
\mu_{y+t, s}^{13} & =f_{2}(s) \cdot \mu_{y+t}^{(2)},
\end{aligned}
$$

where $\psi_{1, x+t}$ and $\psi_{2, y+t}$ are age-dependent parameters and $f_{1}(s)$ and $f_{2}(s)$ are decreasing functions of $s$, the sojourn time in widowhood. We assume that $\psi_{1, x+t}$ and $\psi_{2, y+t}$ are less than one for all ages and $f_{1}(s)$ and $f_{2}(s)$ are greater than one for all $s$. This specification is analogous to the Cox proportional hazards model. The marginal forces of mortality $\mu_{x+t}^{(1)}$ and $\mu_{y+t}^{(2)}$ serve as baseline hazard rates. Proportions $\psi_{1, x+t}$ and $\psi_{2, y+t}$ are less than one to ensure that the forces of mortality in married status are lower than the marginal forces of mortality. $f_{1}(s)$ and $f_{2}(s)$ control the bereavement effect. They are greater than one but asymptotically approach one as $s$ goes to infinity. The bereavement effect initially increases the force of mortality of the surviving spouse and then tapers off as the sojourn in widowhood continues.

The specification of proportional mortality rates is supported by empirical data analysis. Using the data of joint life annuities from a large Canadian insurance company, Ji et al. (2011) and Spreeuw and Owadally (2013) showed that the mortality in married status is significantly lower than the corresponding mortality in widowhood. Spreeuw and Owadally (2013) therefore followed Denuit and Cornet (1999) and specified that the mortality of those who are married is lower than the marginal force of mortality by a constant proportion. In our proposed model, we relax this constraint and permit the proportion to be gender- and age-dependent.

With regard to the mortality in widowhood, Denuit and Cornet (1999) assumed that the "broken-heart" syndrome, which proportionally increases mortality after bereavement, 
persists throughout the entire widowhood. In other words, they assumed that mortality depends on marital status but not on the sojourn time in either married or widowed status and therein contains the Markov property. Spreeuw and Owadally (2013) retained the Markov property for mortality in married status but extended Denuit and Cornets (1999) model by introducing two states of widowhood: early widowhood and ultimate widowhood. The excess mortality in the early widowhood is higher than that in the ultimate widowhood; that is, mortality in widowhood has the semi-Markov property allowing the fading of the "broken-heart" syndrome effect. However, it is difficult to clearly define the threshold between the two post-bereavement states. Scarcity of data has exacerbated this problem and made the model vulnerable to the definition of different widowhood states.

The fading of the bereavement effect has been confirmed by Parkes et al. (1969) based on a statistical study, in which widowers of age 55 and older were followed up for nine years. These authors found that the mortality of widowers increased during the first six months of bereavement and then gradually returned to approximately the same level of married men at the same age. Ji et al. (2011) used an exponential proportional function to govern the initial increase in mortality caused by the bereavement effect and the speed at which the bereavement effect fades. Spreeuw and Owadally (2013) argued that a reverse sigmoid curve with a flatter slope in early widowhood is an alternative choice. Using the reverse sigmoid curve, the speed at which the bereavement effect diminishes is initially slow; however, there is no sufficient data to justify the fading of the bereavement effect. In this paper, we test three different functions for $f_{i}(s)$ to govern mortality deterioration due to bereavement and the speed at which bereavement tapers off. We choose $f_{i}(s)$ to be one of the following three functions:

- an exponential function: $f_{i}(s)=A_{i} e^{-B_{i} s}+1$,

- a Gaussian curve: $f_{i}(s)=A_{i} e^{-\frac{s^{2}}{2 B_{i}^{2}}}+1$, and

- a reverse sigmoid function: $f_{i}(s)=\frac{A_{i}}{1+e^{B_{i}\left(s-C_{i}\right)}}+1$.

Under both exponential function and Gaussian function, the force of mortality at the death of a spouse is $100 A_{i} \%$ above the marginal force of mortality. The exponential function is decreasing and concave up, and as time $s$ increases, the multiplicative factors rapidly decrease and approach 1. The Gaussian function is decreasing but concave downward when $s<B_{i}$, indicating that the multiplicative factor decrease slowly at the early stage of bereavement. When $s \geq B_{i}$, the Gaussian function is concave upward and finally approaches 1. The multiplicative factors decrease at a faster rate when $s$ is close to $B_{i}$ and bereavement effect eventually disappears as $s$ continues to increase. Under a reverse sigmoid function, 
the force of mortality initially increases to slightly less than $100 A_{i} \%$ above the marginal force of mortality. The multiplicative factors decrease very slowly to $50 A_{i} \%$ at $s=C_{i}$ at a rate of $\frac{1}{4} A_{i} B_{i}$, and then gradually approaches 1 .

\subsection{Model Calibration}

It is ideal to calibrate the parameters of the proposed semi-Markov joint life model using bivariate mortality data; however, the only data available for dependent modelling of coupled lifetimes is significantly outdated. The outdated data, with an observation period from December 21, 1988 to December 21, 1993, have been used by Frees et al. (1996), Ji et al. (2011), and Spreeuw \& Owadally (2013). To circumvent the data problem, we use an approximation method to calibrate the model. We match the marginal future lifetime distributions from the model, computed as the mixture of conditional distribution, to the marginal distributions from a chosen annuity life table. The mixture weights can be determined from empirical mortality data if available or inferred from census data.

Let ${ }_{t} p_{x}^{\text {married }}$ be the survival probabilities of a currently married $x$-year-old male. Assume his wife is $y$ years old. The survival probabilities of the husband conditional on the survival of his wife is computed as follows, based on the semi-Markov model and Kolmogorov's forward equation:

$$
\begin{aligned}
{ }_{t} p_{x}^{\text {married }} & =e^{-\int_{0}^{t} \psi_{1, x+s} \mu_{x+s}^{(1)}+\psi_{2, y+s} \mu_{y+s}^{(2)} d s} \\
& +\int_{0}^{t} e^{-\int_{0}^{s} \psi_{1, x+u} \mu_{x+u}^{(1)}+\psi_{2, y+u} \mu_{y+u}^{(2)} d u} \psi_{2, y+s} \mu_{y+s}^{(2)} e^{-\int_{0}^{t-s} f_{1}(u) \mu_{x+s+u}^{(1)} d u} d s
\end{aligned}
$$

To compute the survival probability of a currently widowed person, the time of being widowed is very important due to the semi-Markov property. We therefore categorize the widowed population into $n+1$ groups, for which $w_{s}$, where $s=1,2,3, \ldots, n$, represents the percentage of the population in the $s^{t h}$ year of widowhood and $w_{n+1}$ represent the population who have being widowed for more than $n$ years. We categorize those being widowed for more than $n$ years into one group and assume they experience marginal mortality rates associated with the marginal lifetime distribution. In other words, we assume the bereavement effect diminishes after $n$ years.

Let $\rho_{x}$ represent the percentage of the married population and $\varrho_{x}=\sum_{s=1}^{n+1} w_{s}$ represent the total percentage of the widowed population. Assume that other population groups experience the marginal mortality. Based on the mixture distribution, ${ }_{t} \hat{p}_{x}$, the marginal survival probability of a male at age $x$, can be approximated by solving the following 
equation:

$$
{ }_{t} \hat{p}_{x}=\sum_{s=1}^{n}\left(w_{s} e^{-\int_{0}^{t} f_{1}(s-0.5+u) \mu_{x+u}^{(1)} d u}\right)+\rho_{x} \cdot{ }_{t} p_{x}^{\text {married }}+\left(1-\rho_{x}-\varrho_{x}+w_{n+1}\right){ }_{t} \hat{p}_{x}
$$

The solution to this equation is

$$
{ }_{t} \hat{p}_{x}=\frac{1}{\rho_{x}+\varrho_{x}-w_{n+1}}\left(\rho_{x} \cdot{ }_{t} p_{x}^{\text {married }}+\sum_{s=1}^{n} w_{s} e^{-\int_{0}^{t} f_{1}(s-0.5+u) \mu_{x+u}^{(1)} d u}\right) .
$$

Here we assume that the average time of being widowed in the $s^{\text {th }}$ year of widowhood is $s-0.5$ years for $s=1,2,3, \ldots, n$. The marginal survival probability of a female can be approximated similarly.

We use the proposed model estimation method to calibrate the semi-Markov model to the current pension annuity table, the RP-2014 mortality tables ${ }^{1}$ published by the Society of Actuaries (SOA). We obtain death probability $q_{x+t}$ from the reference table and determine the marginal forces of mortality using the following approximations:

$$
\mu_{x+t}^{(1)} \approx-\ln \left(1-q_{x+t}\right) \quad \text { and } \quad \mu_{y+t}^{(2)} \approx-\ln \left(1-q_{y+t}\right) .
$$

The approximations assume constant force of mortality between integer ages. Parameters of the semi-Markov model are then estimated by minimizing the sum of the relative errors (SRE) between the marginal survival probabilities computed in Equation (2.6) and the survival probabilities from the referenced life table. Expressed Mathematically,

$$
S R E=\sum_{t=1}^{\omega-x}\left(\frac{\left|{ }_{t} p_{x}-{ }_{t} \hat{p}_{x}\right|}{{ }_{t} p_{x}}+\frac{\left|{ }_{t} p_{y}-{ }_{t} \hat{p}_{y}\right|}{{ }_{t} p_{y}}\right),
$$

where $x$ and $y$ denote the age of the male and female respectively, $\omega$ is the limiting age, and ${ }_{t} p_{x}$ and ${ }_{t} p_{y}$ are the male and female survival probabilities obtained from the reference table. Since we focus on the mortality rates at ages 60 and above for annuity valuation, we use $x=60$ and $y=60$ in the calibration. Considering the reliability of the mortality rates in a life table, we use a limiting age of 105 .

The RP-2014 mortality tables were applied to annuity valuation in year 2014 in the US market. Therefore, we also use the Census Bureau's population estimates data for the 55-64 years old age group in $2014^{2}$. The proportions of married and widowed males are approximately $67.4 \%$ and $2.5 \%$. The proportions of married and widowed females are approximately $60.5 \%$ and $7.7 \%$.

\footnotetext{
${ }^{1}$ The abbreviation "RP" represents Retirement Plans.

${ }^{2}$ Source: US Census Bureau, 2014 American Community Survey 1-Year Estimates. Retrieved from https://factfinder. census.gov/faces/tableservices/jsf/pages/productview. xhtml?src=bkmk.
} 
We need to further determine $w_{s}$ for both genders. Since $w_{1}$ represents the percentage of population in the first year of widowhood, it is closely related to the age of the spouse. Suppose that the age difference between a couple is between -5 and 5 . We set $w_{1}$ to 0.005450 for widowers and 0.008011 for widows, which are the average death probabilities of females and males in the age range of 55 to 65 . Since adult mortality increases almost exponentially with age, we assume the percentage of widowers and widows is an exponentially decreasing function of the time being widowed and $w_{s}=w_{1} e^{-k(s-1)}$ for $s=1,2, \ldots, n$. When a person has been in widowhood for more than $n$ years, bereavement effect diminishes and there could be a high chance of remarriage in the census data. We simply assume that the percentage of being widowed for more than $n$ years is $w_{n+1}=w_{1} e^{-k n}$. To determine $k$, we solve $\sum_{s=1}^{n+1} 0.005450 e^{-k(s-1)}=2.5 \%$ for males, and $\sum_{s=1}^{n+1} 0.008011 e^{-k(s-1)}=7.7 \%$ for females. Since the bereavement effect diminishes in less than 10 years under our calibrated models, we let $n$ equal to 10 and obtain $k=0.2219$ for males and $k=0.02775$ for females.

Table 1 lists the parameters estimates of the three functions for $f_{1}(s)$ in Equations $(2.3)$ and $f_{2}(s)$ in Equation (2.4). Figure 2 displays the fitted functions of $f_{1}(s)$ and $f_{2}(s)$ in panel (b) and the estimated age-dependent multiplicative parameters $\psi_{1, x+t}$ and $\psi_{2, y+t}$ in panel (a).

\begin{tabular}{|r|r|r|r|r|r|r|}
\hline \multirow{2}{*}{ Parameter } & \multicolumn{2}{|c|}{ Exponential } & \multicolumn{2}{c|}{ Gaussian } & \multicolumn{2}{c|}{ Sigmoid } \\
\cline { 2 - 7 } & Male & Female & Male & Female & Male & Female \\
\hline$A$ & 7.9475 & 7.9821 & 0.9329 & 0.7689 & 1.9670 & 1.1180 \\
$B$ & 4.6485 & 4.8671 & 1.9374 & 1.9086 & 1.5230 & 1.5789 \\
$C$ & & & & & 4.6851 & 4.2002 \\
\cline { 2 - 7 } Least relative error & \multicolumn{3}{|c|}{$3.3869 \times 10^{-7}$} & \multicolumn{2}{|c|}{$2.9767 \times 10^{-7}$} & \multicolumn{2}{c}{$3.5816 \times 10^{-6}$} \\
\hline
\end{tabular}

Table 1: Parameter of the three functions controlling the bereavement effect using US data

The three functions controlling the bereavement effect all yield favorable fitting errors, although the Gaussian function has slightly lower fitting error than the other two functions. However, the large difference in the estimated $A$ in Table 1 indicates that the three functions model very different extent of "broken-heart" effect at the moment of bereavement. The exponential function results in a sharp initial increase in mortality at the death of a spouse, while both the Gaussian function and the reverse sigmoid function have a much more moderate initial increase.

The lower panels of Figure 2 depict how the bereavement effect fades with time under the three different bereavement effect assumptions. The exponential function has the fastest fading speed, and the reverse sigmoid function gives the slowest fading speed. The upper panels of Figure 2 depict the estimated multiplicative factors for married status using the 

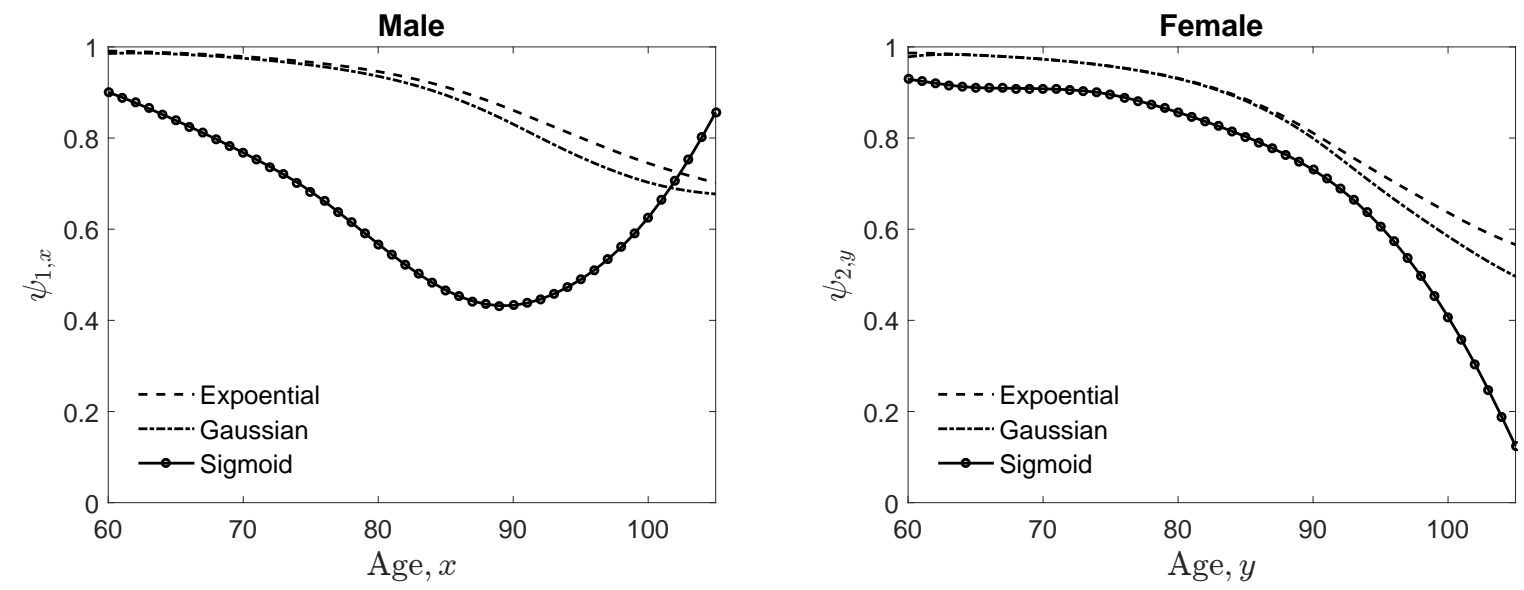

(a) Age-dependent multiplicative parameters for mortality in married status
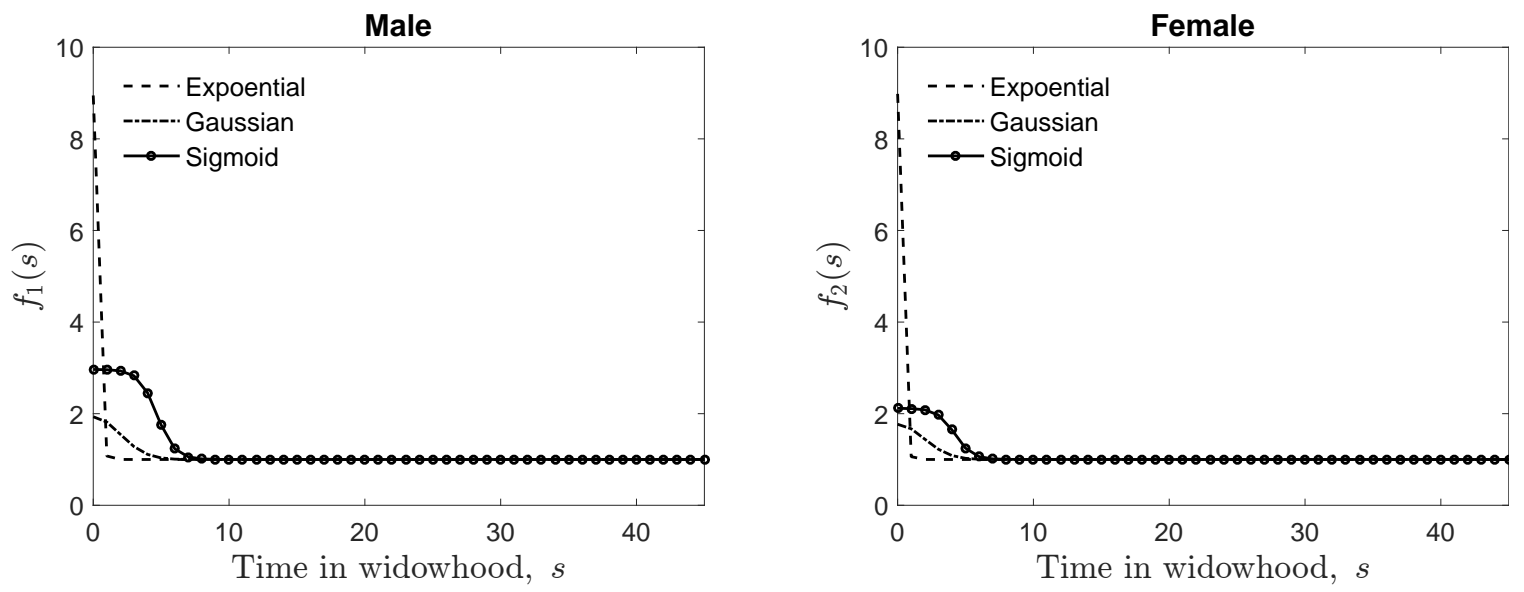

(b) Time-dependent multiplicative functions for mortality in widowed status

Figure 2: Multiplicative factors for the semi-Markov joint-life mortality model using the US RP-2014 life table.

three different bereavement effect assumptions. The exponential function and the Gaussian function result in similar curves of multiplicative factors for mortality in married status. The positive effect of married status on mortality increases with age using both functions. In contrast, using the reverse sigmoid function, we observe a u-shaped pattern for the multiplicative factors in married status for males, with the lowest values around age 95 . The multiplicative factor for high age females in married status using the reverse sigmoid function is much lower than using other functions.

We acknowledge that although the Gaussian function has the smallest sum of relative calibration errors, it is difficult to justify which controlling function is the best without 
sufficient joint life mortality data. Our research focuses on demonstrating the flexibility of the Markovian approaches and the comparison of different bereavement assumptions, rather than choosing the best function for controlling the bereavement effect.

In Appendix A1, we display the results of calibrating the semi-Markov joint life mortality model to the S2 Series of Mortality Tables released by the Continuous Mortality Investigation (CMI) in the UK. Similar to the results of using the US life table, the exponential function has the highest initial mortality increase at the death of a spouse and the fastest speed at which the bereavement effect fades with time. The reverse sigmoid functions has the slowest fading speed with moderate "broken-heart" effect. The Gaussian function is close to the reverse sigmoid function in terms of initial increase and persistence of "broken-heart" effect.

The Gaussian function again has the smallest relative fitting errors using the UK life table. The estimated multiplicative factors for married status using the exponential function and the reverse sigmoid function are similar with those obtained from the US RP-2014 life table. However, the multiplicative factors using Gaussian function are significantly different from the corresponding values obtained from the US life table. In particular, the multiplicative factors for married females are more in line with those from the reverse sigmoid function.

Our proposed calibration method has the merit of circumventing the use of joint life data; however, we acknowledge that the calibration results are dependent on the choice of the function controlling bereavement effect and the assumption of population distribution in different marital status. Practitioners can calibrate the proposed model to their annuitant mortality data if available, and obtain more accurate estimates.

\section{$2.3 \quad$ Implications}

The semi-Markov joint life model captures both short-term and long-term dependence between coupled lifetimes. Short-term dependence reflects the increase in the mortality of the surviving spouse at bereavement and the fading of the bereavement effect over time. Long-term dependence represents the consistently lower mortality of a married couple than the general population. Using this model, the mortality of the surviving spouse increases significantly, especially in the first couple years of bereavement. Taking a 60 -year-old male and a 60-year-old female as an example, Figure 3 illustrates the mortality at bereavement using the exponential, Gaussian, and reverse sigmoid controlling functions respectively. The solid line represents the marginal forces of mortality determined from the RP-2014 Life Table.

In Figure 3, we observe notable mortality deterioration during bereavement, especially 
for males. Ignoring the dependence between coupled lifetimes will lead to the use of the marginal mortality and hence underestimation of mortality in bereavement. In addition, different functions used for the bereavement effect lead to dramatically different patterns of mortality deterioration. If a reverse sigmoid function is used for controlling the bereavement effect, the force of mortality of a 60-year-old male increases to 0.0231 at the death of his spouse, a large jump compared to the marginal force of mortality of 0.0078 from the RP-2014 Life Table. The "broken-heart" effect persists for 8 years and then gradually vanishes over the next couple of years. The same pattern is evident in female mortality at bereavement, although to a much lower extent. The Gaussian function results in a lower "broken-heart" effect which smoothly and gradually tapers off in 7 years. Using the exponential function, mortality deterioration is the highest at the death of a spouse. However, the bereavement effect diminishes after 2 years, the shortest among the three functions.
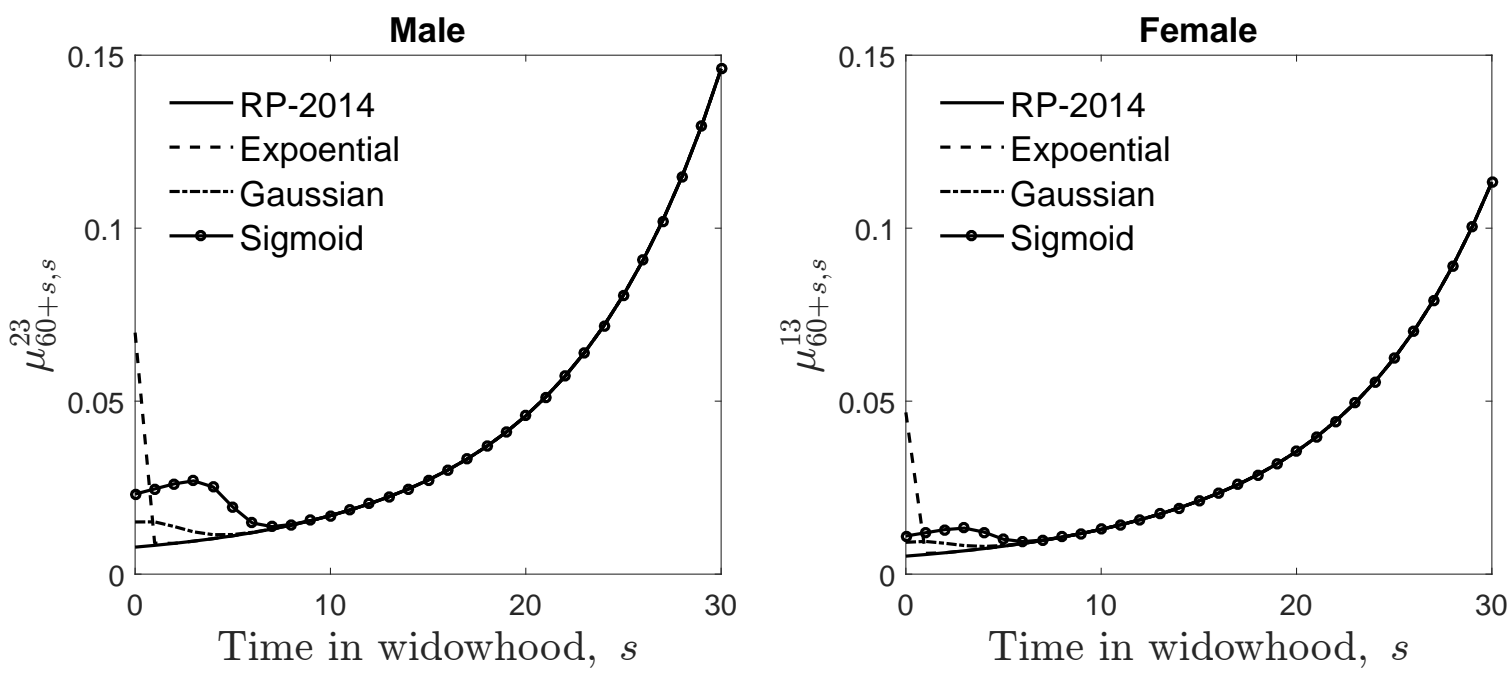

Figure 3: Force of mortality of males and females widowed at age 60 in the US.

In Figure 4, we plot the forces of mortality of married males and females under the three different functions. The unconditional marginal mortality rates observed from the SOA RP-2014 Life Table are plotted in solid line as comparison. Although the exponential function and the Gaussian function result in very different patterns of bereavement effect, they share similar patterns of age-dependent multiplicative factors in married status. The reverse sigmoid function results in the lowest multiplicative factors in married status among the three functions. More interestingly, the mortality of married females using the reverse sigmoid function even decreases with age for age 100 and higher, due to the sharp decrease in multiplicative factors for married females at extremely high ages.

The survival probabilities of coupled lives are therein different from those based on an 

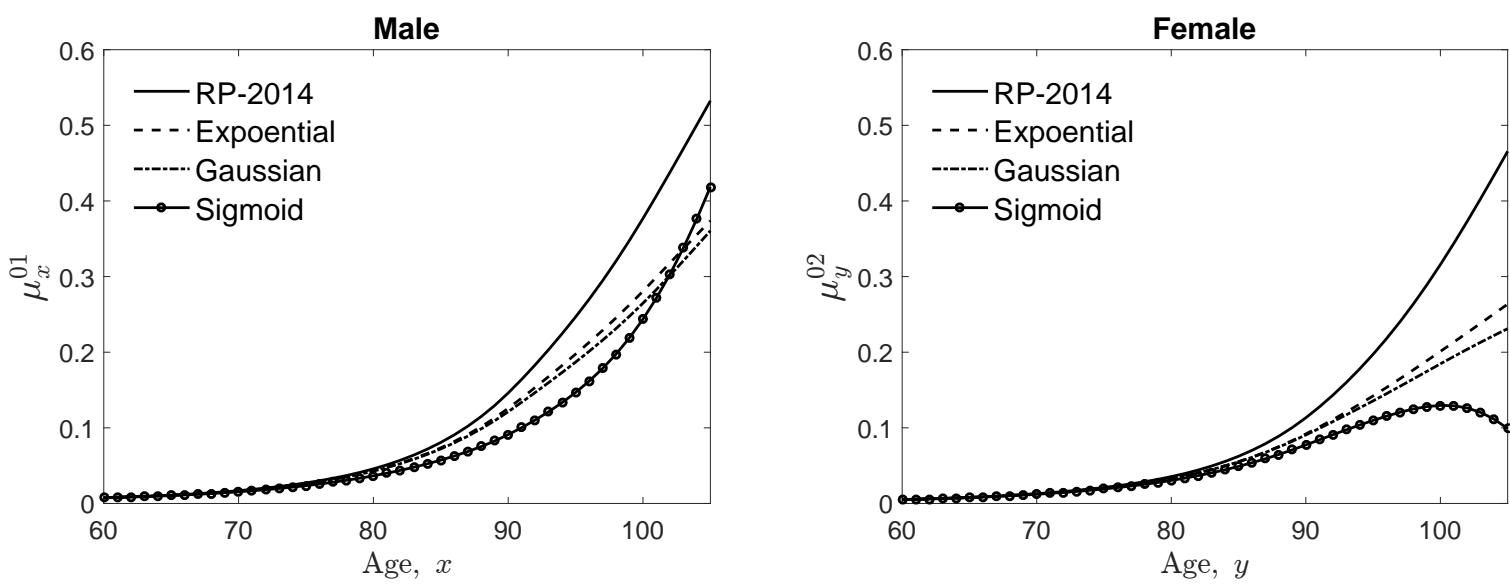

Figure 4: Forces of mortality of married males and females under the three bereavement effect assumptions.

independence model. Ignoring mortality improvement over years for now, the probability of both coupled lives $(x)$ and $(y)$ surviving $t$ years can be computed as

$$
{ }_{t} p_{x y}=\operatorname{Pr}\left[\min \left(T_{x}, T_{y}\right)>t\right]=e^{-\int_{0}^{t}\left(\psi_{1, x+s} \mu_{x+s}^{(1)}+\psi_{2, y+s} \mu_{y+s}^{(2)}\right) d s,}
$$

and the probability that at least one of the couple is alive in $t$ years can be calculated as

$$
\begin{aligned}
{ }_{t} p_{\overline{x y}}= & \operatorname{Pr}\left[\max \left(T_{x}, T_{y}\right)>t\right]={ }_{t} p_{x y}^{00}+{ }_{t} p_{x y}^{01}+{ }_{t} p_{x y}^{02} \\
= & e^{-\int_{0}^{t}\left(\psi_{1, x+s} \mu_{x+s}^{(1)}+\psi_{2, y+s} \mu_{y+s}^{(2)}\right) d s}+ \\
& \int_{0}^{t} e^{-\int_{0}^{s}\left(\psi_{1, x+u} \mu_{x+u}^{(1)}+\psi_{2, x+u} \mu_{x+u}^{(2)}\right) d u} \psi_{2, y+s} \mu_{y+s}^{(2)} e^{-\int_{0}^{t-s} f_{1}(u) \mu_{x+s+u}^{(1)} d u} d s+ \\
& \int_{0}^{t} e^{-\int_{0}^{s}\left(\psi_{1, x+u} \mu_{x+u}^{(1)}+\psi_{2, x+u} \mu_{x+u}^{(2)}\right) d u} \psi_{1, x+s} \mu_{x+s}^{(1)} e^{-\int_{0}^{t-s} f_{2}(u) \mu_{y+s+u}^{(2)} d u} d s
\end{aligned}
$$

Here $(x y)$ is referred to as joint life status and $(\overline{x y})$ is referred to as last survivor status.

$\mu_{x+s}^{(1)}$ and $\mu_{y+s}^{(2)}$, where 1 is for males and 2 for females, are the marginal forces of mortality and can be obtained from the chosen life table.

Different bereavement controlling functions will lead to different projected survival probabilities of coupled lives. In the following application, we use the Gaussian function for controlling the bereavement effect, due to its reasonable calibration results and relatively low fitting error.

\section{Two-population Mortality Improvement Model}

The life tables and implied forces of mortality for married couples are deterministic and do not allow for mortality changes in the future, while the consensus is that mortality 
has improved in the past and will continue to improve in an uncertain way in the future. The higher than expected future mortality improvement is referred to as longevity risk. In fact, longevity risk places enormous strain on social security systems, retirement funds, and private annuity providers. Therefore, we further propose to incorporate mortality improvement to the semi-Markov model.

We use the Age-Period-Cohort (APC) model for modelling mortality improvement. First proposed by Osmond (1985), the APC model is the simplest stochastic mortality model that incorporates cohort effect, the phenomenon that people born in certain years have experienced faster mortality improvement than others. Cohort effect is particularly important for accurate valuation of annuity liabilities. Cairns et al. (2011) found that the APC model produced biologically plausible mortality forecasts and is reasonably robust to the historical data.

Using the APC model, the central mortality rates $m_{x, t}^{(i)}$ is modeled as follows,

$$
\ln \left(m_{x, t}^{(i)}\right)=\alpha_{x}^{(i)}+n_{a}^{-1} \kappa_{t}^{(i)}+n_{a}^{-1} \gamma_{t-x}^{(i)}
$$

where $x$ is age, $t$ is year, $i=1$ for males and 2 for females, $n_{a}$ is the number of observed ages, and $\alpha_{x}, \kappa_{t}, \gamma_{t-x}$ are the age, period, and cohort effects. We assume $D_{x, t}$, the number of death at age $x$ in year $t$, follows a Poisson distribution with mean equal to $m_{x, t} E_{x, t}$, where $E_{x, t}$ is number of exposures-to-risk. Parameters are estimated by the maximum likelihood estimation method with identifiability constraints $\sum_{t} \kappa_{t}^{(i)}=0, \sum_{x, t} \gamma_{t-x}^{(i)}=0$, and $\sum_{x, t} \gamma_{t-x}^{(i)}(x-\bar{x})=0$.

Let $t_{0}$ be the base year of the chosen life table. Assuming that the annuitants will experience the same mortality improvement as experienced by the general population, mortality rates in the life table can be projected into the future year $t$ by the following formula,

$$
\begin{aligned}
\mu_{x, t}^{(i)} & =\mu_{x, t_{0}}^{(i)} \frac{m_{x, t}^{(i)}}{m_{x, t_{0}}^{(i)}} \\
& =\mu_{x, t_{0}}^{(i)} \frac{e^{\alpha_{x}^{(i)}+n_{a}^{-1} \kappa_{t}^{(i)}+n_{a}^{-1} \gamma_{t-x}^{(i)}}}{e^{\alpha_{x}^{(i)}+n_{a}^{-1} \kappa_{t_{0}}^{(i)}+n_{a}^{-1} \gamma_{t_{0}-x}^{(i)}}} \\
& =\mu_{x, t_{0}}^{(i)} \cdot e^{n_{a}^{-1}\left(\kappa_{t}^{(i)}-\kappa_{t_{0}}^{(i)}\right)+n_{a}^{-1}\left(\gamma_{t-x}^{(i)}-\gamma_{t_{0}-x}^{(i)}\right)} .
\end{aligned}
$$

According to the RP - 2014 Mortality Tables Report (2014), the RP-2014 life tables are the mortality rates in the base year 2014 and hence $t_{0}=2014$. If we use S2 Series of Mortality Tables, the base year is $t_{0}=2007$.

In Equation (3.2), age effect parameters are not needed for projecting $\mu_{x, t}^{(i)}$. In addition, to apply the proposed model to new annuitants above age 65 in 2017, we need cohort 
parameters up to the birth year of 1952. Therefore, we use the US and UK population mortality data in the age range of 55 to 95 from year 1963 to 2013 to fit the APC model. After obtaining the parameter estimates for $\kappa_{t}^{(i)}$ and $\gamma_{t-x}^{(i)}$, we further model $\kappa_{t}^{(i)}$ by multivariate time series model.

Besides the dependence between husband and wife, we also consider the correlation between male and female mortality improvement. Li et al. (2015) illustrated a systematic process for constructing two-population mortality models, based on widely used singlepopulation models. They also imposed non-divergence conditions to ensure that mortality rates of two correlated populations will not diverge indefinitely for biological reasonableness. Ji and Zhou (2017) found whether or not to impose the non-divergence conditions has insignificant impact on the value of an immediate annuity. Therefore, we simply use one APC model for each population without imposing the non-divergence constraints.

The estimated parameters are presented in panel (a) of Figure 5 . Both $\kappa_{t}^{(1)}$ and $\kappa_{t}^{(2)}$ decrease almost linearly. The best-fitted model for individual period effect is $\operatorname{ARIMA}(0,1,0)^{3}$ based on Bayesian Information Criteria. Therefore, we use a bivariate random walk with drift to model $\left\{k_{t}^{(1)}, k_{t}^{(2)}\right\}$ :

$$
\left(\begin{array}{c}
\kappa_{t}^{(1)} \\
\kappa_{t}^{(2)}
\end{array}\right)=\left(\begin{array}{l}
d^{(1)} \\
d^{(2)}
\end{array}\right)+\left(\begin{array}{c}
\kappa_{t-1}^{(1)} \\
\kappa_{t-1}^{(2)}
\end{array}\right)+\left(\begin{array}{c}
\epsilon_{t}^{(1)} \\
\epsilon_{t}^{(2)}
\end{array}\right),
$$

where $d^{(1)}$ and $d^{(2)}$ are drift terms, and $\left\{\epsilon_{t}^{(1)}, \epsilon_{t}^{(2)}\right\}$ follows a bivariate normal distribution with zero mean and variance-covariance matrix of $\Sigma_{\epsilon^{(1)} \epsilon^{(2)}}$.

In panel (b) of Figure 5, we display the mean forecast and $90 \%$ prediction intervals of $\kappa_{t}^{(1)}$ and $\kappa_{t}^{(2)}$ for both correlated and independent case. The correlated case uses the bivariate random walk, while the uncorrelated case sets the correlation of the error term in the bivariate random walk to zero. There is minimal difference between the correlated and uncorrelated cases in terms of mean forecast and prediction interval because incorporating correlation does not affect the mean value or the variance of $k_{t}^{(1)}$ and $k_{t}^{(2)}$. Correlation plays an important role when we deal with a mix of population (1) and (2) and it is particular important for pricing annuities. We will revisit this point in last survivor annuity pricing. In Appendix A2, we display parameter estimates and predictions of $\kappa_{t}^{(1)}$ and $\kappa_{t}^{(2)}$ using England and Wales population mortality data.

Suppose that the marginal force of mortality is constant between integer ages, and the transition from state 0 to 1 or state 0 to 2 only occurs at mid year. Taking into account of mortality improvement, the $k$-year last survivor probability of a couple $(x)$ and $(y)$ computed

\footnotetext{
${ }^{3}$ ARIMA stands for Autoregressive Integrated Moving Average model.
} 

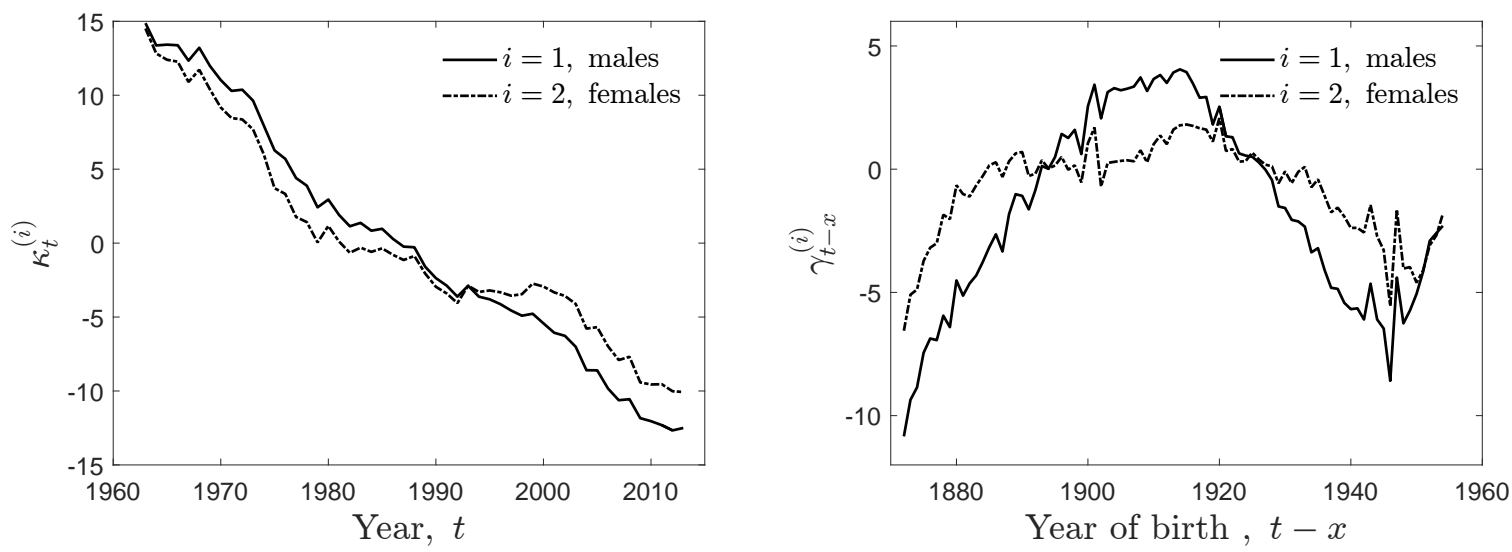

(a) Estimated parameters
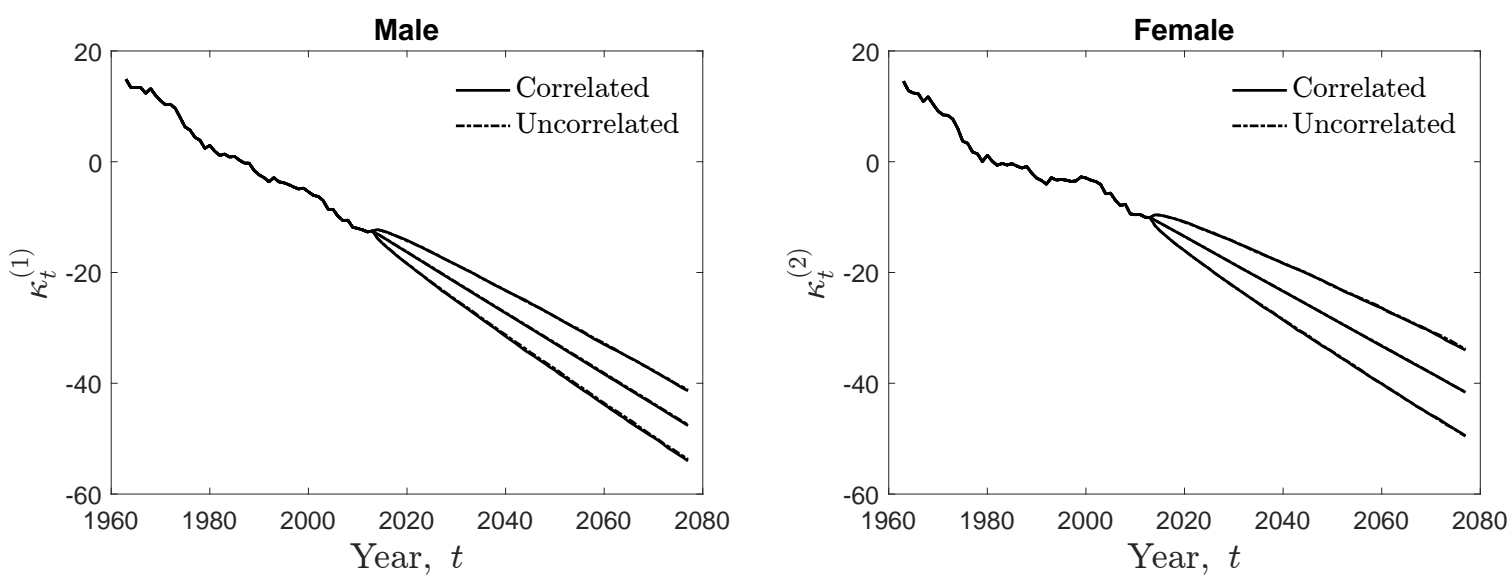

(b) Forecasted $\kappa_{t}^{(1)}$ and $\kappa_{t}^{(2)}$

Figure 5: Panel (a): Estimated $\kappa_{t}^{(i)}$ and $\gamma_{t-x}^{(i)}$ for $t=1963,1964, \ldots, 2013$ and $x=$ $55,56, \ldots, 95$; Panel (b): Mean forecasts and $90 \%$ prediction intervals of $\kappa_{t}^{(1)}$ and $\kappa_{t}^{(2)}$ for $t=2014,2015, \ldots$, using the US population data

in year $T$, can be rewritten as

$$
\begin{aligned}
& { }_{k} p_{\overline{x y}, T}=e^{-\sum_{s=0}^{k-1}\left(\psi_{1, x+s} \mu_{x+s, T+s}^{(1)}+\psi_{2, y+s} \mu_{y+s, T+s}^{(2)}\right)}+ \\
& \sum_{u=0}^{k-1} u+0.5 p_{x y} \cdot \psi_{2, y+u} \mu_{y+u, T+u}^{(2)} \cdot e^{-\left(\int_{0}^{0.5} f_{1}(s) \mu_{x+u, T+u}^{(1)} d s+\sum_{v=1}^{k-1-u} \int_{v-0.5}^{v+0.5} f_{1}(s) \mu_{x+u+v, T+u+v}^{(1)} d s\right)}+ \\
& \sum_{u=0}^{k-1} u+0.5 p_{x y} \cdot \psi_{1, x+u} \mu_{x+u, T+u}^{(1)} \cdot e^{-\left(\int_{0}^{0.5} f_{2}(s) \mu_{y+u, T+u}^{(2)} d s+\sum_{v=1}^{k-1-u} \int_{v-0.5}^{v+0.5} f_{2}(s) \mu_{y+u+v, T+u+v}^{(2)} d s\right)} \cdot
\end{aligned}
$$

We assume the multiplicative factors $\psi_{1, x}$ and $\psi_{2, y}$ and the controlling functions $f_{1}(s)$ and $f_{2}(s)$ will not change over time. This means that both the bereavement effect and agedependent ratios of the forces of mortality in the married status to the marginal forces of 


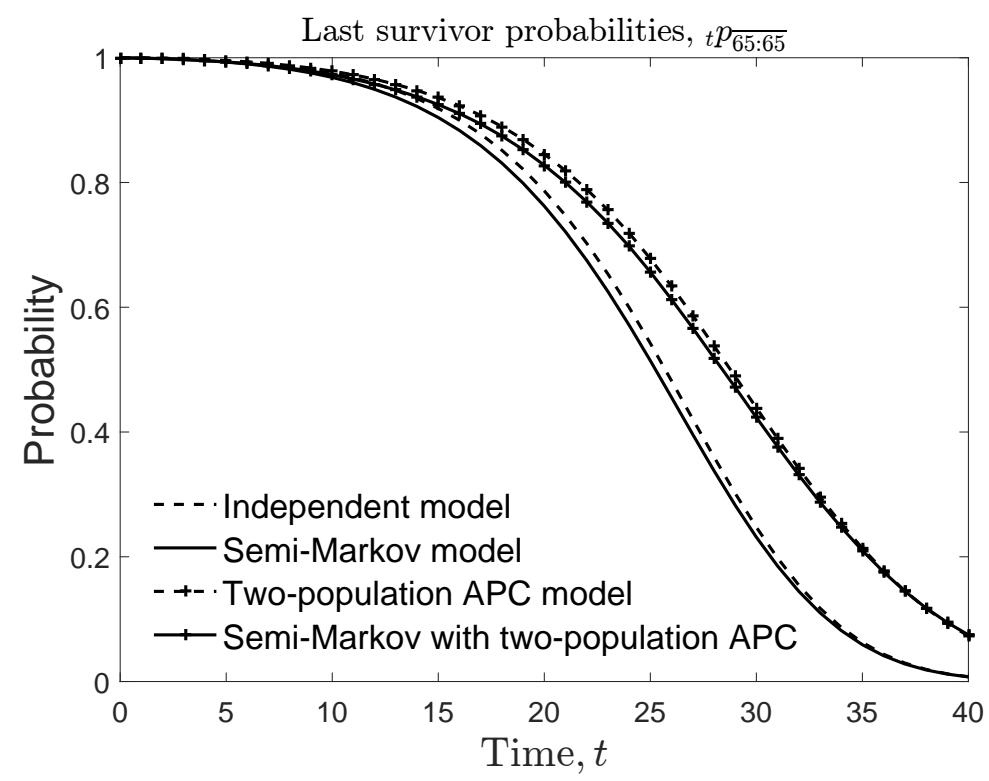

Figure 6: Last survivor probabilities under various dependence assumptions.

mortality do not evolve, although marginal mortality improves over time. These assumptions are realistic, especially when there is no historical mortality data of coupled annuitants to support the claim that the "broken-heart" syndrome changes over time.

Figure 6 displays the survival functions of the last survivor status for a 65 -year-old husband and wife under various dependence assumptions. The solid line uses the semimarkov model to capture the dependence from marital status; and the dashed line assumes independence. Both lines assume no mortality improvement. Comparing the solid and dashed line, we can see that the dependence from marital status causes the last survivor probability to shift downward slightly in 10 to 25 years, due to the bereavement effect in early widowhood. The solid line with "+" marker incorporates the dependence from both marital status and common mortality improvement factors by using the semi-Markov model with mortality improvement. The dashed line with "+" marker only models the correlated mortality improvement by using the two-population APC model. Incorporating mortality improvement shifts survival function upward. The lower survival probability caused by bereavement effect can still be observed by comparing these two lines.

\section{$4 \quad$ Pricing Longevity Risk in Annuities}

In this section we apply the semi-Markov joint life model with mortality improvement to evaluate last survivor annuities sold in the US and UK markets. Based on the proposed 
model and the current quotes of single life annuities and last survivor annuities, we compute the implied market prices of longevity risk in various annuities.

Assuming no arbitrage, the price of a contingent claim is evaluated as the discounted expected payoff under a risk-neutral probability measure. However, there is an infinite number of risk-neutral probability measures in an incomplete market. We further assume that market players act in an equilibrium setting and such an equilibrium setting selects a market consistent risk-neutral measure. The market price of risk is therein implicitly derived from the "risk-neutralized" statistical distribution of a contingent claim written on the underlying risks.

To price a contingent claim on multiple risks, Wang (2007) proposed the usage of multivariate exponential tilting as a general approach of changing the multivariate probability measure. Cox et al. (2006) employed multivariate exponential tilting to derive the implied market price of mortality risk based on the observed price of the Swiss Re bond. We follow this method and examine the implied risk premium of longevity risk in the current US and UK private annuity markets.

\subsection{Normalized Multivariate Exponential Tilting}

Definition 1. For each scenario $\omega$ in the probability space $(\Omega, \mathcal{F}, P)$, the exponential tilting of $X_{1}, X_{2}, \ldots, X_{n}$ with respect to references $Y_{1}, Y_{2}, \ldots, Y_{k}$ is defined by the following probability density function:

$$
\frac{f^{*}\left(x_{1}, x_{2}, \ldots, x_{n}\right)}{f\left(x_{1}, x_{2}, \ldots, x_{n}\right)}=c \cdot E\left[\exp \left(\sum_{j=1}^{k} \lambda_{j} Y_{j}\right) \mid X_{1}=x_{1}, X_{2}=x_{2}, \ldots, X_{n}=x_{n}\right],
$$

where $\lambda_{j}$ 's are real-valued parameters controlling the magnitude of risk adjustment, and $c$ is a normalizing coefficient.

The flexibility in the choice of the references $Y_{1}, Y_{2}, \ldots, Y_{n}$ gives an inconsistent interpretation of the parameter $\lambda$. In order to have a meaningful interpretation of $\lambda$, Wang (2007) proposed the application of a normalization procedure to the reference variable as defined below.

Definition 2. Assume that there exist standard normal variables $\left\{Z_{1}, Z_{2}, \ldots, Z_{k}\right\}$, such that

$$
Y_{1}=F_{Y_{1}}^{-1}\left(\Phi\left(Z_{1}\right)\right), Y_{2}=F_{Y_{2}}^{-1}\left(\Phi\left(Z_{2}\right)\right), \ldots, Y_{k}=F_{Y_{n}}^{-1}\left(\Phi\left(Z_{k}\right)\right) .
$$

The normalized multivariate exponential tilting of $\left\{X_{1}, X_{2}, \ldots, X_{n}\right\}$ with respect to refer- 
ences $\left\{Y_{1}, Y_{2}, \ldots, Y_{k}\right\}$ is defined as:

$$
\frac{f^{*}\left(x_{1}, x_{2}, \ldots, x_{n}\right)}{f\left(x_{1}, x_{2}, \ldots, x_{n}\right)}=c \cdot E\left[\exp \left(\sum_{j=1}^{k} \lambda_{j} Z_{j}\right) \mid X_{1}=x_{1}, X_{2}=x_{2}, \ldots, X_{n}=x_{n}\right],
$$

where $\Phi(\cdot)$ is the cumulative distribution function of a standard normal variable.

Wang (2007) proved that the normalized multivariate exponential tilting of $X_{1}, X_{2}, \ldots, X_{n}$ with respective normalized reference variables $Z_{1}, Z_{2}, \ldots, Z_{k}$ is equivalent to the multivariate Wang transform, as stated in the following theorem.

Theorem 1. Assume that $\left\{X_{1}, X_{2}, \ldots, X_{n} ; Y_{1}, Y_{2}, \ldots, Y_{k}\right\}$ follow a normal copula. The normalized multivariate exponential tilting of $\left\{X_{1}, X_{2}, \ldots, X_{n}\right\}$ with respect to references $\left\{Y_{1}, Y_{2}, \ldots, Y_{k}\right\}$ is equivalent to applying Wang transforms to $X_{i}$ with

$$
F_{X_{i}}^{*}\left(x_{i}\right)=\Phi\left[\Phi^{-1}\left(F_{X_{i}}\left(x_{i}\right)\right)-\eta_{i}\right], \text { and } \eta_{i}=\Sigma_{j=1}^{k} \rho_{X_{i} Y_{j}} \cdot \lambda_{j},
$$

where $\rho_{X_{i} Y_{j}}$ is the correlation coefficient of $X_{i}$ and $Y_{j}$.

In the APC mortality model, $\left\{\kappa_{t}^{(1)}, \kappa_{t}^{(2)}\right\}$ are modeled by bivariate random walk with drift. Assume that $\left\{\epsilon_{t}^{(1)}, \epsilon_{t}^{(2)}\right\}$ use themselves as references. Using the bivariate exponential tilting, the transformed error term under the risk-neutral measure is

$$
\left(\begin{array}{c}
\epsilon_{t}^{(1)^{*}} \\
\epsilon_{t}^{(2)^{*}}
\end{array}\right)=\left(\begin{array}{c}
\epsilon_{t}^{(1)}-\eta_{1} \sigma_{1} \\
\epsilon_{t}^{(2)}-\eta_{2} \sigma_{2}
\end{array}\right)
$$

where $\eta_{i}=\sum_{j=1}^{2} \rho_{\epsilon_{t}^{(i)} \epsilon_{t}^{(j)}} \lambda_{j}$ and $\sigma_{i}$ is the standard deviation of $\epsilon_{t}^{(i)}$.

Using market consistent risk-neutral survival probabilities, a last survivor annuity is evaluated as the actuarial present value of annuity payments discounted at the risk-free interest rate. The implied market prices of longevity risk, $\lambda_{1}$ and $\lambda_{2}$, are derived by equating the actuarial present value to the quoted market prices.

Let $\ddot{a}_{\overline{x y}}(2017)$ denote the market value quoted in 2017 of a last survivor annuity-due with $\$ 1$ per year paid in advance. Under the risk-neutral measure, the last survivor annuity-due issued to coupled lives $(x)$ and $(y)$ can be expressed as

$$
\ddot{a}_{\overline{x y}}(2017)=1+\sum_{t=1}^{\omega-\min (x, y)-1} \frac{1}{(1+i)^{t}} E_{Q\left(\lambda_{1}, \lambda_{2}\right)}\left[{ }_{t} p_{\overline{x y}} \mid \mathcal{F}_{0}\right]
$$

where $i$ is the annual effective risk-free interest rate, and $E_{Q\left(\lambda_{1}, \lambda_{2}\right)}[$.$] is the expectation$ under the risk-neutral measure defined by the risk adjustment parameters $\lambda_{1}$ and $\lambda_{2}$. The price of an annuity with more frequent payments can be approximated assuming uniform 
distribution of deaths between integer ages. For instance, the price of an annuity-due with monthly payments can be approximated by $\ddot{a}_{\overline{x y}}^{(12)}=\frac{i d}{i^{(12)} d^{(12)}} \ddot{a}_{\overline{x y}}-\frac{i-i^{(12)}}{i^{(12)} d^{(12)}}$, where $d$ is the annual effective discount rate, $i^{(12)}$ is the nominal interest rate compounded monthly, and $d^{(12)}$ the nominal discount rate compounded monthly.

\subsection{Implied Joint-life Longevity Risk Premium in the US}

Last survivor annuities with different combinations of inception ages might not be consistently priced; therefore, we quote a series of last survivor annuities to form a picture of the market's view of joint life longevity risk. The risk-free interest rate is set as $i=2.78 \%$, which is the average yield rate of the US 20-year treasury bills in the first quarter of 2017 according to the daily yield curve rates data ${ }^{4}$.

We quote the last survivor annuity rates from the ImmediateAnnuity.com. The quoted annuity rate is the monthly annuity payment starting one month after the quote date per $\$ 100,000$ of premium at issue. For last survivor annuities, the level regular payments continue until the last survivor dies. The expense loading is $4 \%$, as assumed by Cox and Lin (2007). The monthly annuity payment, $P$, can be obtained by solving the following equation:

$$
12(1.04) P a \frac{(12)}{x y}=\$ 100,000,
$$

where $a_{\overline{x y}}^{(12)}$ is the value of an immediate annuity of $\$ 1$ per year with monthly instalment and $a_{\overline{x y}}^{(12)}=\ddot{a}_{\overline{x y}}^{(12)}-\frac{1}{12}$. $\ddot{a}_{\overline{x y}}^{(12)}$ is calculated using Equation (4.4) with UDD approximation.

We assume that the annuitant and his or her spouse are of the same age. Table 2 lists the quoted annuity rates for different age combinations. Single life annuity rates are also quoted for comparison. From the quotes we observe that annuity rates depend on both gender and inception ages in the US annuity market, differing from the gender-neutral price rule in the UK. The monthly payments of the last survivor annuities are lower than those of the individual single life annuities, because the last survivor status is expected to last longer than the survival of each individual. The single life annuity rates for males are higher than those for females at the same age because females have higher life expectancy. This differs from the gender-neutral price rule in the UK.

Using the quoted last survivor annuities, we derive the market prices of longevity risk by equating the annuity payment obtained from Equation (4.5) to the market quotes. Since we have one quote of last survivor annuity payment for each age combination but two unknowns $\left(\lambda_{1}\right.$ and $\left.\lambda_{2}\right)$ to solve, there is no unique solution. Therefore, we assume $\lambda_{1}=\lambda_{2}$ in the calibration to stipulate a unique solution.

\footnotetext{
${ }^{4}$ Source: US Department of Treasury.
} 


\begin{tabular}{ccccc}
\hline Male age & Female age & Single-male & Single-female & Last survivor \\
\hline 65 & 65 & $\$ 533$ & $\$ 506$ & $\$ 449$ \\
66 & 66 & $\$ 547$ & $\$ 517$ & $\$ 457$ \\
67 & 67 & $\$ 561$ & $\$ 528$ & $\$ 467$ \\
68 & 68 & $\$ 577$ & $\$ 541$ & $\$ 477$ \\
69 & 69 & $\$ 594$ & $\$ 556$ & $\$ 489$ \\
70 & 70 & $\$ 611$ & $\$ 572$ & $\$ 498$ \\
71 & 71 & $\$ 630$ & $\$ 587$ & $\$ 510$ \\
72 & 72 & $\$ 654$ & $\$ 606$ & $\$ 523$ \\
73 & 73 & $\$ 676$ & $\$ 623$ & $\$ 538$ \\
74 & 74 & $\$ 702$ & $\$ 648$ & $\$ 555$ \\
75 & 75 & $\$ 725$ & $\$ 669$ & $\$ 571$ \\
\hline
\end{tabular}

Table 2: Monthly payments in arrears per $\$ 100,000$ of premium at issue for single life and last survivor annuities quoted in April 2017 for US annuitants.

For consistence, we also assume $\lambda_{1}=\lambda_{2}$ when calibrating the market prices of risk for single life annuities. Since we have two single life annuity quotes, one for males and the other for females, we minimize the sum of pricing errors for single life male and female annuities in the calibration. The pricing error is defined as the square of the difference between the annuity payment obtained from Equation (4.5) and the market quote.

Panel (a) and (b) of Figure 7 depict the implied market prices of longevity risk in last survivor annuities using different dependence assumptions. Panel (a), based on the semiMarkov dependence structure with correlated mortality improvements for coupled lives, assumes dependence from both marital status and common mortality improvement factors. Panel (b) assumes only the correlation of mortality improvement between males and females.

Surprisingly, we observe that the implied market prices of longevity risk in both panels are negative. If annuity providers were compensated for bearing the longevity risk, we should expect positive market prices of risk. One explanation for the negative risk premium is that annuity providers may have predicted much slower mortality improvement than the APC model used in this paper. The market prices of risk are forced to be negative and the future lifetime distribution is shifted to the left, in order to match the risk-neutral annuity payment with the quoted rate. The extent of underestimation in mortality improvement is more severe for younger annuitants. This might be due to the cross subsidy between ages or natural hedge within the annuity line of business.

Comparing panel (a) and (b) of Figure 7, we observe that ignoring the dependence from 

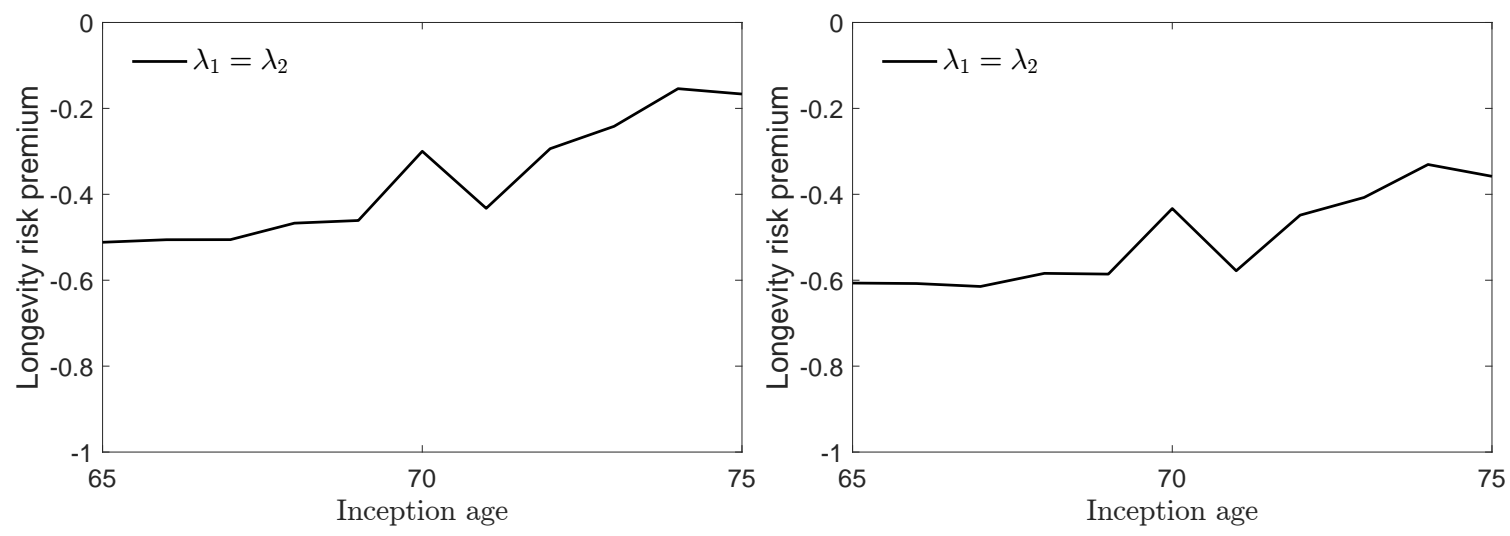

(a) Last survivor, semi-Markov model with mor-

(b) Last survivor, two-population APC model tality improvement

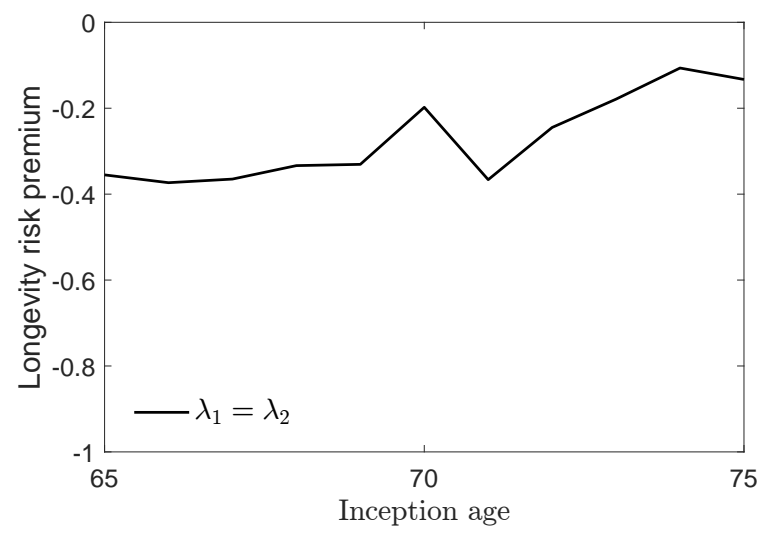

(c) Single life, two-population APC model

Figure 7: Impled market prices of longevity risk in last survivor annuities and single life annuities sold in the US.

marital status leads to more negative markets price of risk. Recall that in Figure 6, using the two-population APC model results in higher survival probability and hence faster mortality improvement than using the semi-Markov model with mortality improvement. Therefore, when using the two-population APC model, more risk adjustment is needed to match the distribution implied by the quoted rates. The difference between the market prices of risk under the two models is more obvious for older annuitants. Since older annuitants have higher chances of being in widowhood, the impact of modelling bereavement effect is more significant.

To further understand the market's view of longevity risk, we examine the market prices of single life longevity risk in panel (c) of Figure 7. Single life mortalities are projected using the two-population APC model. Comparing panel (a) and (c), the implied market prices of 
risk in single life annuities are generally less negative than those in last survivor annuities. It suggests that there is less underestimation of mortality improvement in single life annuities.

Overall, underpricing exists in both last survivor and single life annuities, and it is more severe for last survivor annuities sold to younger annuitants. Our findings are consistent with Ji (2011). The use of semi-Markov model for the dependence due to marital status yields a lower survival probability and slightly offsets the underpricing problem. However, conventional pricing practice often ignores this dependence and hence suffers more from underpricing.

\subsection{Implied Joint life Longevity Risk Premium in the UK}

The UK market has a different pricing practice. In March 2011, the European Court of Justice ruled that it is unlawful for European Union member states to use gender as a factor in the calculation of insurance premiums and benefits. Thereafter unisex annuity prices took effect on December 21, 2012, meaning that annuity providers have to offer the same annuity prices for both genders in the European market. ${ }^{5}$ The quoted annuity rates in the UK market are based on the age of annuitants or the younger annuitants in the case of joint lives. Therefore, the annuity rates in Table 3 for single life male, single life female, and last survivor annuities are the same for annuitants at the same age. These annuity rates were provided by Hargreaves Lansdown Asset Management on April 24, 2017 based on an average post code, one of the pricing factors in the UK.

The annuity prices in the UK do not reflect the difference between male and female mortality, nor do they reflect the difference between single life and joint life mortality. To calibrate the market prices of longevity risk, we choose a risk-free interest rate of $i=1.82 \%$, which is the average yield rate of 20-year government gilts in the first quarter of 2017 (retrieved from the published daily yield curve $\operatorname{rates}^{6}$ ). We also assume the expense loading is $4 \%$.

Figure 8 demonstrates the implied market prices of risk in single life and last survivor annuities. Panel (a) and (b) are the implied market prices of longevity risk in last survivor annuities based on the semi-Markov model with mortality improvement and the two-population APC model respectively. Similar with the US market, we observe negative market prices of risk, indicating that the last survivor annuities have been significantly underpriced and mortality improvement has been underestimated. Since the semi-Markov model with mortality improvement yields lower survival probability than the two-population

\footnotetext{
${ }^{5}$ While the unisex pricing regulation applies to employer-sponsored pension plans or government pension plans in the US, it is not compulsory in the US private annuity market.

${ }^{6}$ Source: Bloomberg and Bank calculations.
} 


\begin{tabular}{ccccc}
\hline Male age & Female age & Single-male & Single-female & Last survivor \\
\hline 65 & 65 & $£ 443$ & $£ 443$ & $£ 443$ \\
66 & 66 & $£ 458$ & $£ 458$ & $£ 458$ \\
67 & 67 & $£ 473$ & $£ 473$ & $£ 473$ \\
68 & 68 & $£ 490$ & $£ 490$ & $£ 490$ \\
69 & 69 & $£ 508$ & $£ 508$ & $£ 508$ \\
70 & 70 & $£ 528$ & $£ 528$ & $£ 528$ \\
71 & 71 & $£ 550$ & $£ 550$ & $£ 550$ \\
72 & 72 & $£ 576$ & $£ 576$ & $£ 576$ \\
73 & 73 & $£ 604$ & $£ 604$ & $£ 604$ \\
74 & 74 & $£ 634$ & $£ 634$ & $£ 634$ \\
75 & 75 & $£ 666$ & $£ 666$ & $£ 666$ \\
\hline
\end{tabular}

Table 3: Monthly payments in advance per $£ 100,000$ of premium at issue for single life and last survivor annuities quoted in April 2017 for UK annuitants.

APC model, the implied market prices of risk are in panel (a) are less negative. The underpricing issue appears worse in the UK market compared to the US market. In addition, as opposed to the US, there is a downward trend in the implied market prices of risk in the UK as inception age increases.

Panel (c) plots the implied market prices of longevity risk in single life annuities. The implied market prices of risk are close to zero in general, suggesting that single life annuities are fairly priced. Recall that in Table 3, the quoted rates for last survivor annuities are the same with those for single life annuities. However, the life expectancy of the last survivor should be greater than or equal to that of the single life. Forcing the same annuity rates on the two types of annuities will inevitably lead to significant underpricing of last survivor annuities.

\section{Discussion and Conclusions}

In this paper, we propose a general semi-Markov mortality model for dynamic modelling of dependence between coupled lifetimes. Under the proposed model, the force of mortality in married status is lower than the marginal force of mortality, while the force of mortality in widowed status is much higher at early widowhood and gradually approaches the marginal mortality as time elapses. Three different functions are tested for controlling the impact of bereavement effect on mortality caused by the "broken-heart" syndrome. 

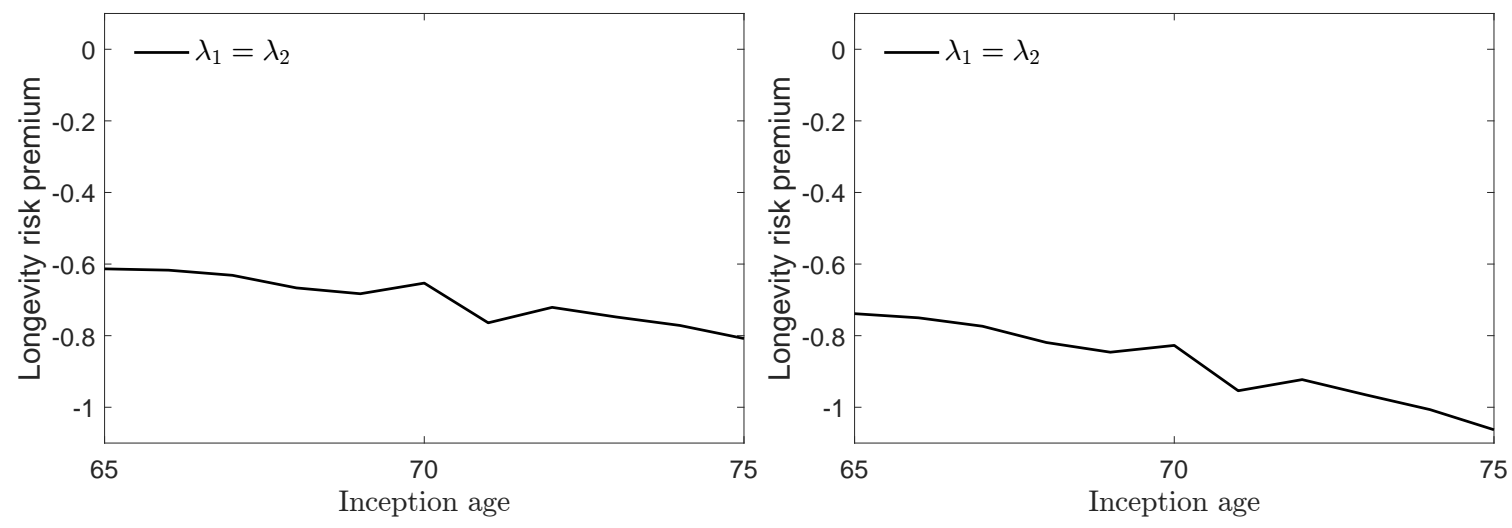

(a) Last survivor, semi-Markov model with mor-

(b) Last survivor, two-population APC model tality improvement

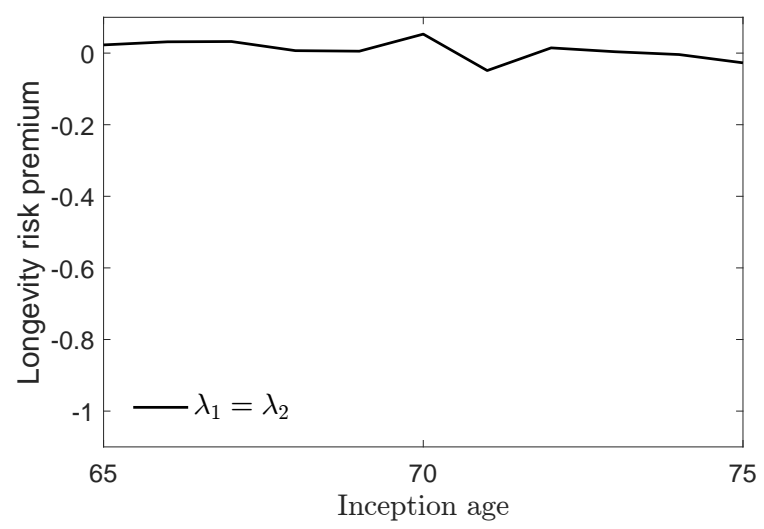

(c) Single life, two-population APC model

Figure 8: Implied market price of longevity risk in last survivor annuities and single-life annuities in the UK.

We also propose a calibration method to circumvent the problem of lack of bivariate mortality data in model estimation. The proposed method matches marginal mortality rates computed from the semi-Markov model with the mortality rates obtained from a chosen life table. We recognize that this calibration method is confined to the chosen life table and the census data of marital status. The calibration of the semi-Markov model will be more accurate if bivariate mortality data are available.

The two-population APC mortality model is incorporated to the semi-Markov model to project future mortality rates. We are interested in the fairness and financial soundness of annuity prices. To this end, we employ the concept of implied market price of longevity risk and investigate the market's view of longevity risk when pricing various annuities sold in the US and UK. The multivariate exponential tilting method is used to distort the distribution 
of underlying risk and derive a market-consistent risk-neutral probability measure.

We find that the implied market prices of longevity risk in both single life and last survivor annuities are negative in the US, indicating that annuities are significantly underpriced. This is likely due to the underestimation of future mortality improvement in practice compared to the two-population APC model used in this study. We also examine the implied market prices of risk with and without considering the dependence from marital status. The bereavement effect captured by the semi-Markov model results in lower survival probabilities for last survivor status, and hence somewhat offsets the impact of underestimation of mortality improvement. The underpricing in last survivor annuities is even more significant in UK, because of the indifferent pricing for single life and last survivor annuities.

The prevalent aggressive pricing practice is possibly due to the stress from the so-called "annuity-puzzle". However, massive negative risk premium of longevity might be a warning signal that the financial soundness of annuity pools and pension plans are jeopardized. In this sense, mortality assumptions for annuity pricing need to be reviewed. Further investigation of annuity fairness is needed, with regard to the unexpected mortality improvement in the future and the financial strain caused by longevity risk.

In addition, the bereavement effect modeled by the proposed semi-Markov model has higher impact on the pricing of reversionary annuities than on last survivor annuities. Since the payments of reversionary annuities start after the death of one spouse, the mortality of the surviving spouse plays a more critical role. Without considering the bereavement effect, one may overestimate the survival probability of the surviving spouse and overprice reversionary annuities.

\section{References}

[1] Cairns, A. J. G., Blake, D., Dowd, K., Coughlan, G. D., \& Khalaf-Allah, M. (2011). Bayesian stochastic mortality modelling for two populations. Astin Bulletin, 41(1), 29-59.

[2] Cairns, A. J., Blake, D., Dowd, K., Coughlan, G. D., Epstein, D., \& Khalaf-Allah, M. (2011). Mortality density forecasts: An analysis of six stochastic mortality models. Insurance: Mathematics and Economics, 48(3), 355-367.

[3] Continuous Mortality Investigation Self-administered Pension Schemes Mortality Committee Working Paper 71. Retrieved from https://www. actuaries.org.uk/learn-and-develop/continuous-mortality-investigation/ cmi-working-papers/saps/cmi-wp-71 
[4] Cox, P., \& Ford, J. (1964). The mortality of widows shortly after widowhood. The Lancet, 283(7325), 163-164.

[5] Cox, S. H., \& Lin, Y. (2007). Natural hedging of life and annuity mortality risks. North American Actuarial Journal, 11(3), 1-15.

[6] Cox, S. H., Lin, Y., \& Wang, S. (2006). Multivariate exponential tilting and pricing implications for mortality securitization. Journal of Risk and Insurance, 73(4), 719736.

[7] Dickson, D. C., Hardy, M. R., \& Waters, H. R. (2013). Actuarial mathematics for life contingent risks. Cambridge University Press.

[8] Denuit, M., \& Cornet, A. (1999). Multilife premium calculation with dependent future lifetimes. Journal of Actuarial Practice, 7, 147-171.

[9] Dowd, K., Cairns, A. J. G., Blake, D., Coughlan, G. D., \& Khalaf-Allah, M. (2011). A gravity model of mortality rates for two related populations. North American Actuarial Journal, 15(2), 334-356.

[10] Frees, E. W., Carriere, J., \& Valdez, E. (1996). Annuity valuation with dependent mortality. Journal of Risk and Insurance, 229-261.

[11] Hubener, A., Maurer, R., \& Rogalla, R. (2013). Optimal portfolio choice with annuities and life insurance for retired couples. Review of Finance, rfs046.

[12] Ji, M., Hardy, M., \& Li, J. S. H. (2011). Markovian approaches to joint-life mortality. North American Actuarial Journal, 15(3), 357-376.

[13] Ji, M., \& Zhou, R. (2017). Demographic risk in deep-deferred annuity valuation. Annals of Actuarial Science, 1-29.

[14] Ji, M. (2011). Markovian approaches to joint life mortality with applications in risk management (Unpublished doctoral dissertation). University of Waterloo, Canada.

[15] Li, J. S. H., \&Hardy, M. R. (2011). Measuring basis risk in longevity hedges. North American Actuarial Journal, 15(2), 177-200.

[16] Li, J. S. H., Zhou, R., \& Hardy, M. (2015). A step-by-step guide to building twopopulation stochastic mortality models. Insurance: Mathematics and Economics, 63, 121-134.

[17] Lin, Y., \& Cox, S. H. (2005). Securitization of mortality risks in life annuities. Journal of Risk and Insurance, 72(2), 227-252. 
[18] Office for National Statistics (2010). Population Estimates by Marital Status, Mid-2002 - Mid-2008. Retrieved from http://webarchive.nationalarchives. gov.uk/20160105160709/http://www.ons.gov.uk/ons/rel/pop-estimate/ population-estimates-by-marital-status/mid-2002-mid-2008/index.html

[19] Osmond, C. (1985). Using age, period and cohort models to estimate future mortality rates. International Journal of Epidemiology, 14(1), 124-129.

[20] Parkes, C. M., Benjamin, B., \& Fitzgerald, R. G. (1969). Broken heart: A statistical study of increased mortality among widowers. Br med $J, 1(5646), 740-743$.

[21] Society of Actuaries RP - 2014 Mortality Tables Report (2014). Retrieved from https://www.soa.org/experience-studies/2014/research-2014-rp/

[22] Spreeuw, J., \& Owadally, I. (2013). Investigating the broken-heart effect: a model for short-term dependence between the remaining lifetimes of joint lives. Annals of Actuarial Science, 7(02), 236-257.

[23] Wang, S. (2007). Normalized exponential tilting: Pricing and measuring multivariate risks. North American Actuarial Journal, 11(3), 89-99.

[24] Young, M., Benjamin, B., \& Wallis, C. (1963). The mortality of widowers. The Lancet, 282(7305), 454-457.

[25] Youn, H., \& Shemyakin, A. (2001). Pricing practices for joint last survivor insurance. Actuarial Research Clearing House, 1(2), 3.

[26] Zhou, R., Wang, Y., Kaufhold, K., Li, J.S.H., \&Tan, K.S. (2014). Modelling mortality of multiple populations with Vector Error Correction Models: Applications to Solvency II. North American Actuarial Journal, 18(1), 150-167.

\section{Appendix A1: : Calibration of the semi-Markov Model to the UK Data}

In this appendix, we list the results of applying the semi-Markov joint life morality model to the UK data. We use the life table released by the Continuous Mortality Investigation (CMI) Self-administered Pension Schemes (SAPS) Mortality Committee. The life table is based on data collected by June 30, 2012, and is referred as "S2 Series of Mortality Tables. This table applies to mortality in year 2007; therefore, we use the UK marital status population estimates in year 2007 from the Population Estimates by Marital Status Mid-2002 - Mid-2008 Report by the Office for National Statistics (2010) to approximate 
$\rho_{60}$ and $\varrho_{60}$ and $w_{s}$ for $s=1,2, \ldots, 11$ in Equation 2.6. Table 4 displays the estimated parameters, and the plots of the three fitted functions are depicted in Panel (b) of Figure 9 .

\begin{tabular}{|c|c|c|c|c|c|c|}
\hline \multirow{2}{*}{ Parameter } & \multicolumn{2}{|c|}{ Exponential } & \multicolumn{2}{c|}{ Gaussian } & \multicolumn{2}{c|}{ Sigmoid } \\
\cline { 2 - 7 } & Male & Female & Male & Female & Male & Female \\
\hline$A$ & 8.3912 & 8.2314 & 2.3599 & 1.3343 & 2.9666 & 1.0331 \\
$B$ & 5.0258 & 4.9775 & 3.1367 & 3.1115 & 1.2314 & 1.2819 \\
$C$ & & & & & 5.0558 & 4.6546 \\
\cline { 2 - 7 } Least relative error & \multicolumn{3}{|c|}{$8.8331 \times 10^{-7}$} & \multicolumn{2}{|c|}{$5.3079 \times 10^{-7}$} & \multicolumn{2}{c|}{$5.6840 \times 10^{-6}$} \\
\hline
\end{tabular}

Table 4: Parameter of the three functions controlling the bereavement effect using UK data

Panel (a) of Figure 9 displays the fitted age-dependent multiplicative factors for forces of mortality in the "Both alive" state of the semi-Markov model. The multiplicative factors for each gender based on the exponential function and reverse sigmoid functions have the same shape as those from the US life table; while the multiplicative factors based on the Gaussian function are slightly different from those calibrated to the US life table.

\section{Appendix A2: Calibrating the APC model using UK Data}

In this appendix, we present in Figure 10 the estimated $\kappa_{t}^{(i)}$ and $\gamma_{t-x}^{(i)}$ of the APC model using the total population data for England and Wales total population data, where $i=1$ for males and 2 for females, for $t=1963,1964, \ldots, 2013$ and $x=55,56, \ldots, 95$. We also demonstrate the mean value and $90 \%$ confidence interval of the projected $\kappa_{t}^{(1)}$ and $\kappa_{t}^{(2)}$ for $t=2014,2015, \ldots, 2078$, assuming $\kappa_{t}^{(1)}$ and $\kappa_{t}^{(2)}$ are correlated and uncorrelated. 

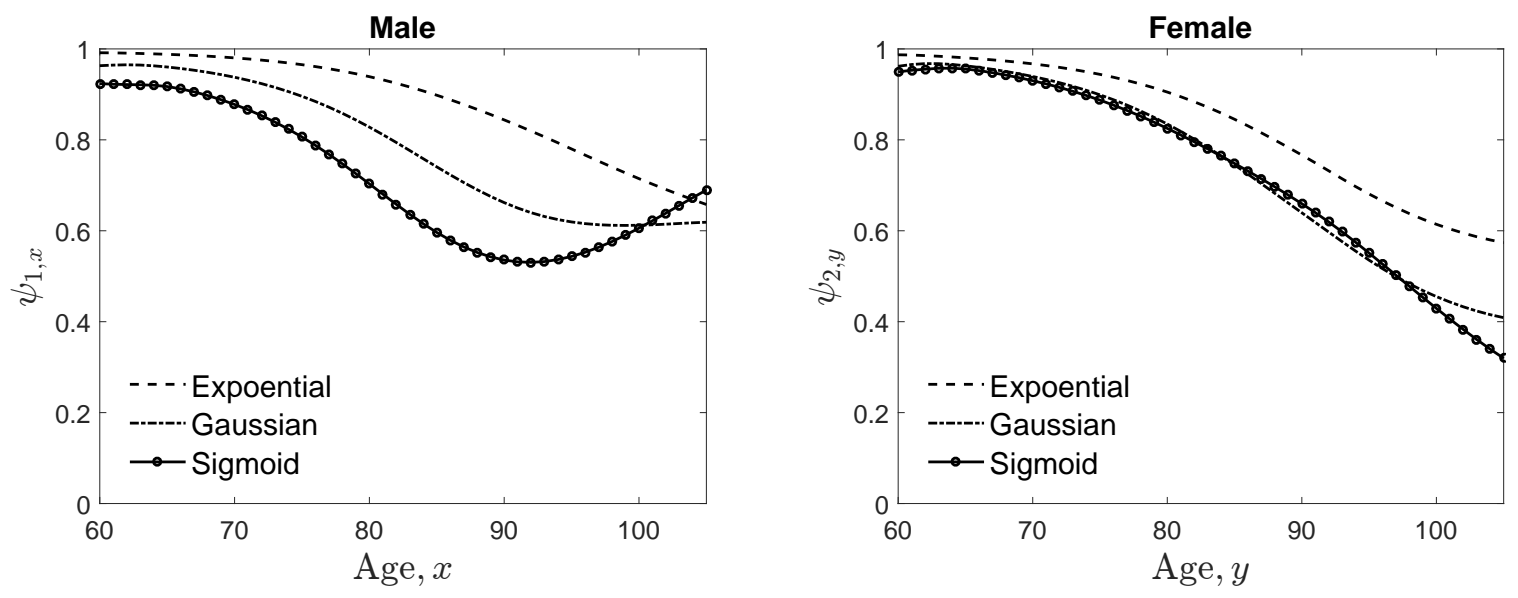

(a) Age-dependent multiplicative parameters for mortality in married status
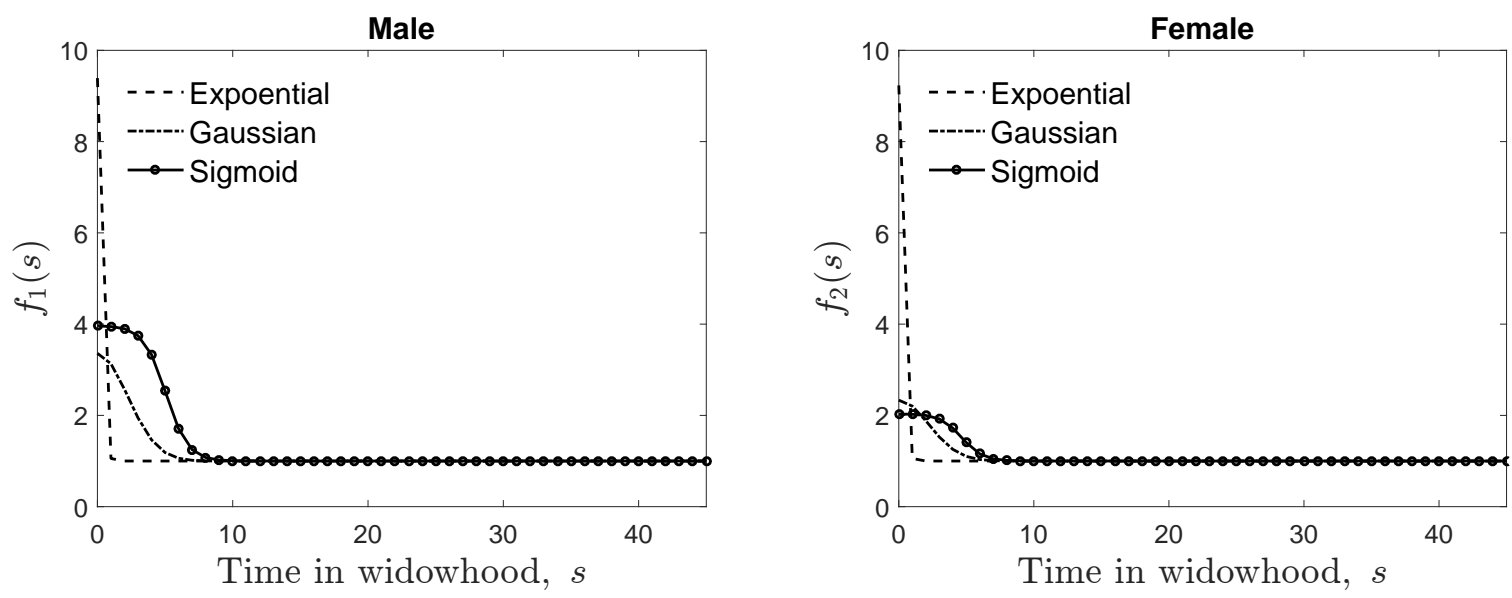

(b) Three time-dependent multiplicative functions for mortality in widowed status

Figure 9: Multiplicative factors for the semi-Markov joint-life mortality model using UK data. 

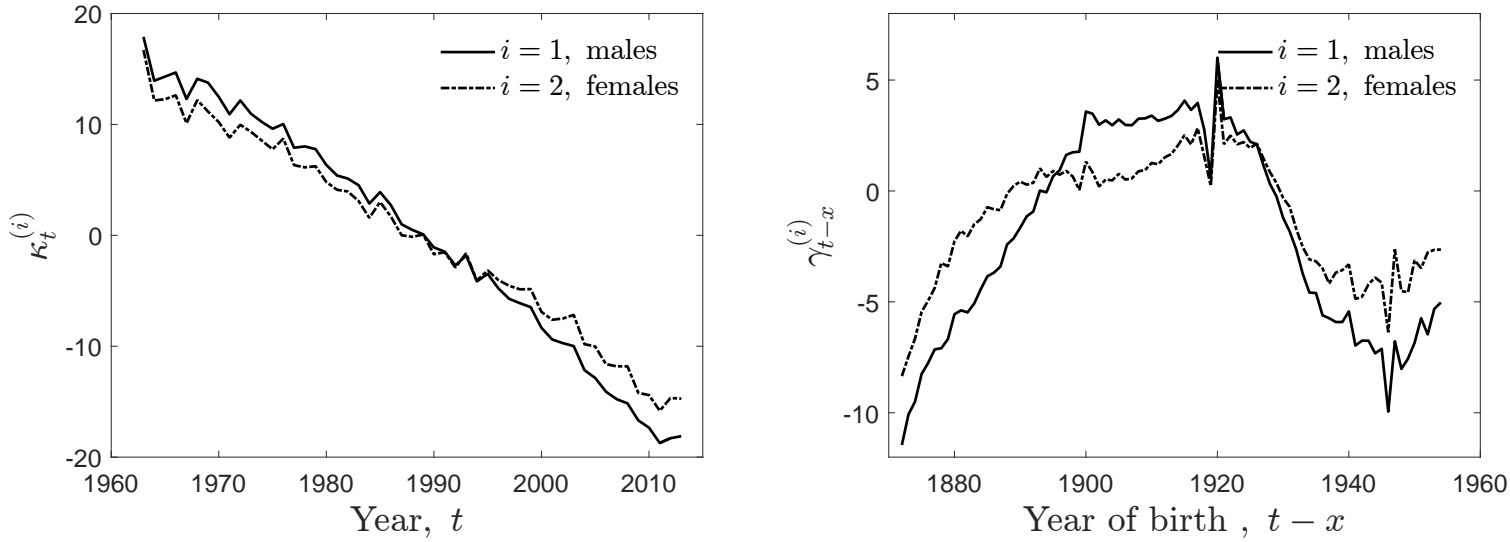

(a) Estimated parameters
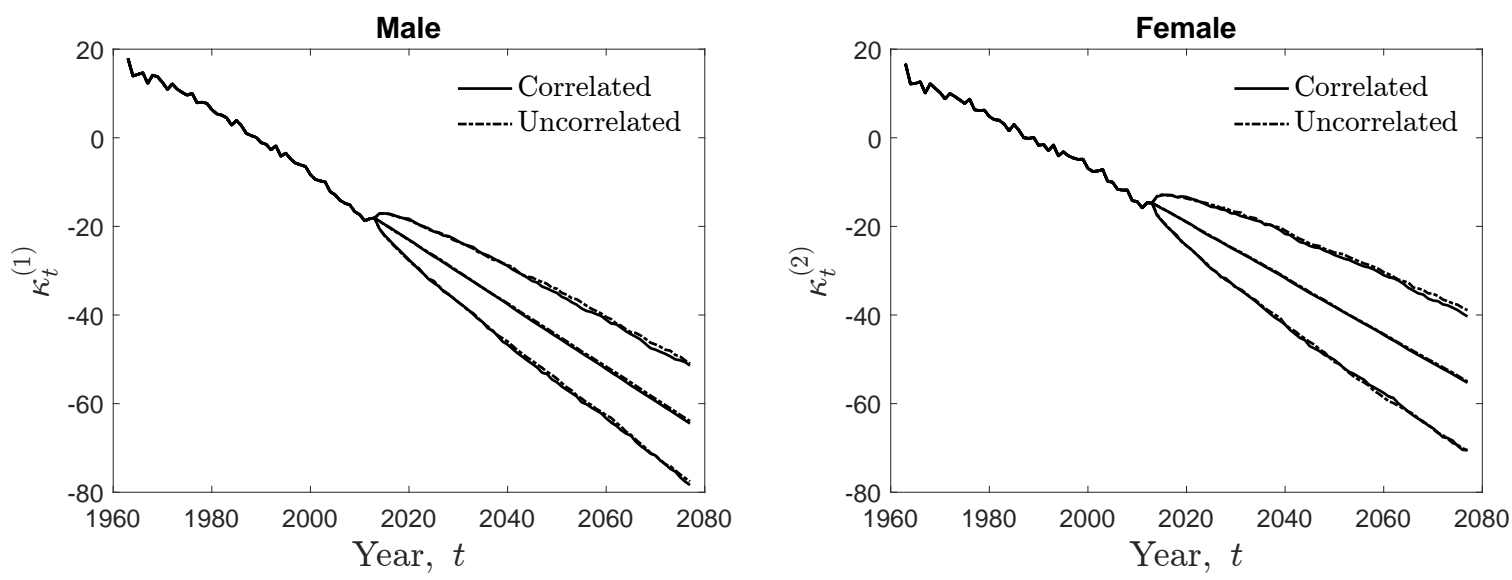

(b) Forecasted $\kappa_{t}^{(1)}$ and $\kappa_{t}^{(2)}$

Figure 10: Estimated $\kappa_{t}^{(i)}$ and $\gamma_{t-x}^{(i)}$ for $t=1963,1964, \ldots, 2013$ and $x=55,56, \ldots, 95$ (upper) using the UK mortality data, and the forecasted $90 \%$ confidence intervals of $\kappa_{t}^{(1)}$ and $\kappa_{t}^{(2)}$ for $t=2014,2015, \ldots 2078$. 